Collision-induced absorption with exchange effects and anisotropic interactions: Theory and application to $\mathrm{H}_{2}-\mathrm{H}_{2}$

Tijs Karman, Ad van der Avoird, and Gerrit C. Groenenboom

Citation: The Journal of Chemical Physics 142, 084305 (2015); doi: 10.1063/1.4907916

View online: http://dx.doi.org/10.1063/1.4907916

View Table of Contents: http://aip.scitation.org/toc/jcp/142/8

Published by the American Institute of Physics

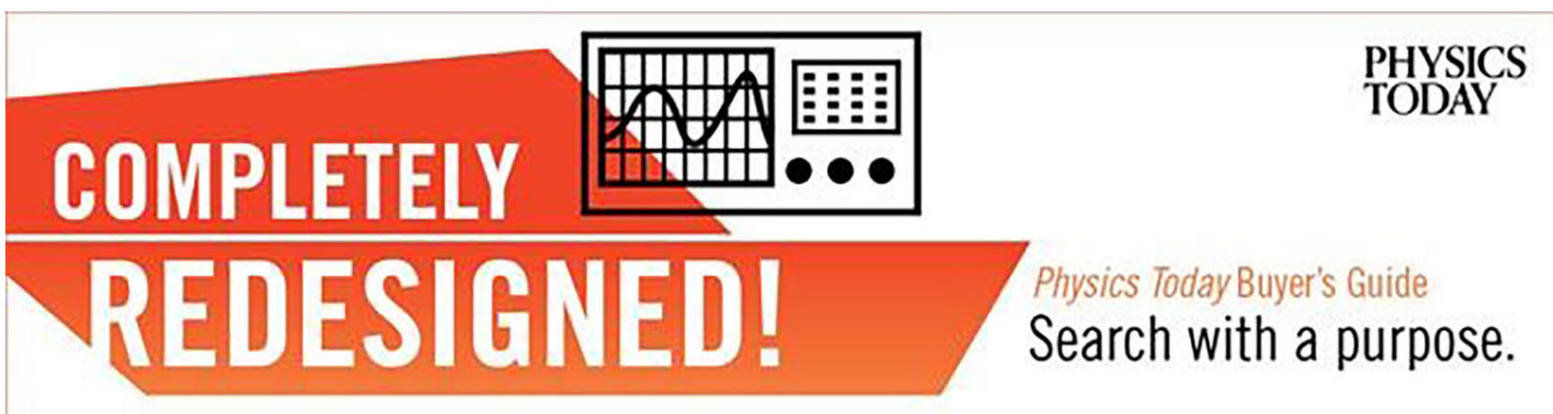




\title{
Collision-induced absorption with exchange effects and anisotropic interactions: Theory and application to $\mathrm{H}_{2}-\mathrm{H}_{2}$
}

\author{
Tijs Karman, Ad van der Avoird, and Gerrit C. Groenenboom ${ }^{\text {a) }}$ \\ Theoretical Chemistry, Institute for Molecules and Materials, Radboud University Nijmegen, Nijmegen, \\ The Netherlands
}

(Received 2 December 2014; accepted 27 January 2015; published online 25 February 2015)

\begin{abstract}
We discuss three quantum mechanical formalisms for calculating collision-induced absorption spectra. First, we revisit the established theory of collision-induced absorption, assuming distinguishable molecules which interact isotropically. Then, the theory is rederived incorporating exchange effects between indistinguishable molecules. It is shown that the spectrum can no longer be written as an incoherent sum of the contributions of the different spherical components of the dipole moment. Finally, we derive an efficient method to include the effects of anisotropic interactions in the computation of the absorption spectrum. This method calculates the dipole coupling on-the-fly, which allows for the uncoupled treatment of the initial and final states without the explicit reconstruction of the many-component wave functions. The three formalisms are applied to the collision-induced rotationtranslation spectra of hydrogen molecules in the far-infrared. Good agreement with experimental data is obtained. Significant effects of anisotropic interactions are observed in the far wing. (C) 2015 AIP Publishing LLC. [http://dx.doi.org/10.1063/1.4907916]
\end{abstract}

\section{INTRODUCTION}

Homonuclear diatomic molecules, which include several astrophysically and atmospherically relevant species, have zero electric dipole moment due to their inversion symmetry. This means that these molecules are in principle infrared inactive. However, gases of apolar molecules exhibit collisioninduced absorption, as was first shown experimentally for a gas of oxygen molecules. ${ }^{1}$ This absorption originates from collision complexes present in the gas. The collision complex can be considered to be a short-lived species, which may have a lower symmetry than the individual molecules and hence an electric dipole moment. Since these collision complexes are short-lived, the resulting absorption spectra are typically broad in frequency.

The hydrogen molecule, $\mathrm{H}_{2}$, is extensively studied in the field of collision-induced absorption. This includes experimental studies, ${ }^{2,3}$ theoretical studies using the isotropic interaction approximation and empirical potentials, ${ }^{4,5}$ similar theoretical methods applied to vibrational transitions, ${ }^{6}$ and calculations using $a b$ initio potentials and dipole moment surfaces up to very high temperatures. ${ }^{7}$ The anisotropy of the interaction potential has been considered in calculations of the dimer contributions, ${ }^{8}$ as well as in continuum transitions. ${ }^{3}$ The importance of the collision-induced spectra of $\mathrm{H}_{2}$ in astrophysics is discussed extensively in a recent review paper, Ref. 9. To name a few applications: the presence of $\mathrm{H}_{2}$ molecules in the atmospheres of outer planets can be probed by collision-induced absorption, ${ }^{10}$ collision-induced emission is a necessary mechanism for rapid cooling during the formation of the first stars, ${ }^{11}$ and the missing infrared spectra of cool white dwarfs are explained by the absorption due to their

\footnotetext{
a)Electronic address: gerritg@ theochem.ru.nl
}

dense hydrogen atmospheres. ${ }^{12}$ In this paper, we focus on the hydrogen molecule precisely because it is extensively studied, which allows us to compare our theoretical developments to experimental results and other theoretical work.

In this paper, we first discuss the established theory of collision-induced absorption, assuming isotropic interactions between distinguishable molecules. The theory is then rederived incorporating exchange effects between indistinguishable molecules. This method treats exchange interactions exactly within the approximation of an isotropic interaction, unlike an earlier approximate method that applied a statistical weighting to different partial waves. ${ }^{4}$ Finally, we outline an efficient novel method for including the effects of anisotropic interactions in the computation of the absorption spectrum. The three formalisms for calculating collision-induced absorption spectra are then applied to molecular hydrogen. Due to the large rotational constant of the hydrogen molecule, few rotational states are thermally populated. Therefore, exchange effects are expected to be comparatively strong for $\mathrm{H}_{2}-\mathrm{H}_{2}$. On the other hand, the large rotational constant effectively renders the hydrogen molecule isotropic. Significant effects of interaction anisotropy are observed only in the far wing of the absorption spectrum. We further benchmark our method for including anisotropic interactions in the companion paper, where we consider absorption due to nitrogen-nitrogen collisions. ${ }^{13}$

The proposed method for including anisotropic interactions in the calculation of collision-induced spectra is not the first approach developed. McKellar and Schäfer calculated the dipole coupling between initial and final states by explicitly calculating the many-component wave function on a discrete radial grid. ${ }^{8}$ This requires a lot of computer memory, and this approach becomes infeasible if the number of channels becomes large. Julienne and Mies used a different 
approach, treating the radiation field non-perturbatively, ${ }^{14}$ and applied this method to atomic transitions. ${ }^{15-17}$ Gustafsson and Frommhold have applied the same non-perturbative approach to molecular systems such as $\mathrm{H}_{2}-\mathrm{H}_{2}$ and $\mathrm{H}_{2}-\mathrm{He}$. ${ }^{3,18}$ One advantage of this approach is that it may also be applied to multi-photon transitions, as the absorption is treated explicitly, rather than by first order perturbation theory. However, this approach requires the coupled treatment of initial and final states, which essentially doubles the size of the channel basis, making the method unnecessarily computer intensive if one is interested in the weak radiation limit. The method of calculating the dipole coupling on-the-fly, proposed in this paper, allows for the uncoupled treatment of the initial and final states in the scattering calculation, whilst avoiding the need to compute the full many-component wave function on a large radial grid. With this method, it becomes possible to study molecular systems with strong anisotropic interactions, which is demonstrated for the case of collision-induced absorption spectra of $\mathrm{N}_{2}-\mathrm{N}_{2}$, considered in a companion paper. ${ }^{13}$

This paper is organized as follows. Section II discusses the three theoretical formalisms: The usual isotropic interaction approximation is revisited in Sec. II B, then the theory is rederived incorporating exchange between identical molecules in Sec. II C, and finally we present an algorithm for treating anisotropic interactions in Sec. II D. In Sec. III, we apply these three formalisms to the collision-induced rotation-translation spectra of molecular hydrogen. We discuss the potential energy and dipole moment surfaces in Sec. III A and the spin statistics of the $\mathrm{H}_{2}-\mathrm{H}_{2}$ collision complex in Sec. III B. Sections III C and III D discuss the computational details of calculations with isotropic and anisotropic interactions, respectively. Finally, we present numerical results in Sec. III E and compare these to experimental data. Concluding remarks are given in Sec. IV.

\section{THEORY}

\section{A. Absorption coefficients from perturbation theory}

Collision-induced absorption may be computed by first order time-dependent perturbation theory. ${ }^{19}$ The absorption coefficient is defined by Lambert's law, which predicts exponential decay of the intensity, $I(z) \propto \exp (-\alpha z)$, with the path length, $z$. In first order perturbation theory, the absorption coefficient at frequency $\omega$ is given by

$$
\alpha(\omega, T)=\frac{2 \pi^{2}}{3 \hbar c} n^{2} \omega\left[1-\exp \left(-\frac{\hbar \omega}{k_{B} T}\right)\right] V g(\omega, T)
$$

with the spectral density

$$
g(\omega, T)=\bigvee_{i} \oint_{f} P^{(i)}(T)|\langle i|\hat{\mu}| f\rangle|^{2} \delta\left(\omega_{f}-\omega_{i}-\omega\right) .
$$

Here, $n, V$, and $T$ are the number density, volume, and temperature of the gas, respectively. The constant $c$ is the speed of light, $\hbar$ is the reduced Planck constant, and $\hat{\mu}$ represents the dipole operator. The states $|i\rangle$ and $|f\rangle$ represent the initial and final states, with energies $\hbar \omega_{i}$ and $\hbar \omega_{f}$, and $P^{(i)}(T)$ is the thermal population of the initial state. The symbol $\&$ denotes a summation over all discrete quantum numbers, such as rotational states, and integration over the continuum, i.e., translational states.

The initial and final states are the eigenstates of the zeroth order Hamiltonian

$$
\hat{H}=-\frac{\hbar^{2}}{2 \mu} \nabla_{R}^{2}+\hat{H}_{A}+\hat{H}_{B}+\hat{V}\left(\vec{r}_{A}, \vec{r}_{B}, \vec{R}\right),
$$

that is, the Hamiltonian describing the molecular pair but not the coupling with the radiation field. The first term represents the kinetic energy in the center of mass frame, $\hat{H}_{A}$ and $\hat{H}_{B}$ are the monomer Hamiltonians, and $\hat{V}\left(\vec{r}_{A}, \vec{r}_{B}, \vec{R}\right)$ represents the interaction between both molecules. The vector $\vec{r}_{A}$ connects the two atoms in molecule $A, \vec{r}_{B}$ is the analogue for molecule $B$, and the vector $\vec{R}$ connects the centers of mass of both molecules. The dependence of $\hat{\boldsymbol{\mu}}$ and $\hat{V}$ on the polar angles of $\vec{r}_{A}, \vec{r}_{B}$, and $\vec{R}$ is discussed in the Appendix for the case of colliding homonuclear diatomic molecules. Section III A discusses how the dipole moment and potential energy surfaces are determined from electronic structure calculations.

The eigenstates of Hamiltonian Eq. (3) can be divided in two parts: the bound states are eigenstates at discrete energies $E<0$, whereas a continuum of scattering states exists at positive energies $E>0$. The absorption contains contributions of transitions between two bound states, between two scattering states, and between bound and scattering states. In this work, we will focus on transitions between scattering states only. The contribution of bound states is discussed in the companion paper. ${ }^{13}$

In what follows, we will consider evaluating the spectral density, Eq. (2), for scattering wave functions determined in different approximations. We will start with assuming isotropic interactions, since in this approximation, the angular part of the scattering wave function is known analytically and only the radial part must be computed numerically. Next, in Sec. II C, these solutions are adapted to exchange symmetry. Finally, in Sec. II D, we allow for mixing of different angular components, induced by anisotropic interactions between the two molecules.

\section{B. Isotropic interactions between distinguishable molecules}

An approximation that historically plays a central role in the computation of collision-induced spectra is the approximation of an isotropic interaction between the colliding particles. In this approximation, one can separate translational and rotational degrees of freedom and write the scattering state in Dirac notation as

$$
\begin{aligned}
\left|\left(N_{A} N_{B}\right) N L ; J M E_{\mathrm{col}}\right\rangle & =\left|\left(N_{A} N_{B}\right) N L ; J M\right\rangle\left|E_{\mathrm{col}}, L\right\rangle, \\
\left\langle R \mid E_{\mathrm{col}}, L\right\rangle & =\frac{1}{R} U_{E_{\mathrm{col}}, L}(R) .
\end{aligned}
$$

Here, the coupled angular function is defined as

$$
\begin{aligned}
\left|\left(N_{A} N_{B}\right) N L ; J M\right\rangle & \sum_{\substack{M_{A}, M_{B}, M_{N}, M_{L}\\
}}\left|N_{A} M_{A}\right\rangle\left|N_{B} M_{B}\right\rangle\left|L M_{L}\right\rangle \\
\quad & \left\langle N_{A} M_{A} N_{B} M_{B} \mid N M_{N}\right\rangle\left\langle N M_{N} L M_{L} \mid J M\right\rangle,
\end{aligned}
$$


where $\left|N_{A} M_{A}\right\rangle$ and $\left|N_{B} M_{B}\right\rangle$ are the monomer rotational states, $\left|L M_{L}\right\rangle$ describes the end-over-end rotation of the complex, and the symbol $\left\langle l_{1} m_{1} l_{2} m_{2} \mid l m\right\rangle$ denotes a Clebsch-Gordan coefficient. The radial part, $U_{E_{\mathrm{col}}, L}(R) / R$, depends only on the collision energy, $E_{\mathrm{col}}$, and the partial wave, $L$. Therefore, in this approximation, we do not need to calculate the radial wave function for each unique set of quantum numbers, and the calculation of the required wave functions constitutes a computationally inexpensive one-dimensional problem, unlike the coupled differential equations that arise in the general theory. Furthermore, since the angular part of the wave functions is known analytically, the summations over certain quantum numbers that arise in the thermal average, Eq. (2), can be performed analytically, as will be shown in this section.

The total energy of the scattering state, Eq. (4), is given by the sum of the kinetic and rotational energy,

$$
\begin{aligned}
E_{\mathrm{tot}} & =E_{\mathrm{col}}+E_{\mathrm{rot}}, \\
E_{\mathrm{rot}} & =E_{\mathrm{rot}, A}+E_{\mathrm{rot}, B} \\
E_{\mathrm{rot}, X} & =B_{0} N_{X}\left(N_{X}+1\right)-D_{0}\left[N_{X}\left(N_{X}+1\right)\right]^{2},
\end{aligned}
$$

where $X=A, B$ and the rotational and distortion constants of hydrogen are given by $B_{0}=59.322 \mathrm{~cm}^{-1}$ and $D_{0}=0.0471$ $\mathrm{cm}^{-1}$, respectively. ${ }^{20}$ The population of the initial state is given by the product of translational and rotational Boltzmann factors,

$$
\begin{aligned}
P^{(i)}(T) & =P_{\text {trans }} P_{N_{A}} P_{N_{B}}, \\
P_{\text {trans }} & =\frac{\lambda_{0}^{3}}{V} \exp \left(-\frac{E_{\mathrm{col}}}{k_{B} T}\right), \\
\lambda_{0} & =\hbar \sqrt{\frac{2 \pi}{\mu k T}}, \\
P_{N_{X}} & =\frac{g_{N_{X}}}{Z_{\mathrm{rot}}} \exp \left(-\frac{E_{\mathrm{rot}, \mathrm{N}_{X}}}{k_{B} T}\right), \\
Z_{\mathrm{rot}} & =\sum_{N}(2 N+1) g_{N} \exp \left(-\frac{E_{\mathrm{rot}, \mathrm{N}}}{k_{B} T}\right),
\end{aligned}
$$

where $X=A, B$ and $\mu$ is the reduced mass of the collision complex. For hydrogen, the statistical weights are $g_{N_{X}}=1$ if $N_{X}$ is even and $g_{N_{X}}=3$ if $N_{X}$ is odd.

Inserting the scattering wave functions as initial and final states into Eq. (2), the spectral density can be expressed as

$$
\begin{aligned}
V g(\omega, T)= & \lambda_{0}^{3} \hbar \sum_{N_{A}, N_{B}, N} \sum_{N_{A}^{\prime}, N_{B}^{\prime}, N^{\prime}} P_{N_{A}} P_{N_{B}} \sum_{L, L^{\prime}} \int_{0}^{\infty} d E_{\mathrm{col}} \\
& \times \int_{0}^{\infty} d E_{\mathrm{col}}^{\prime} \exp \left(-\frac{E_{\mathrm{col}}}{k_{B} T}\right) \\
& \times \sum_{J, J^{\prime}, M, M^{\prime}} \mid\left\langle\left(N_{A} N_{B}\right) N L ; J M E\right| \\
& \times\left.\hat{\mu}\left|\left(N_{A}^{\prime} N_{B}^{\prime}\right) N^{\prime} L^{\prime} ; J^{\prime} M^{\prime} E^{\prime}\right\rangle\right|^{2} \\
& \times \delta\left(E_{\mathrm{tot}}^{\prime}-E_{\mathrm{tot}}-\hbar \omega\right) .
\end{aligned}
$$

The scattering states are energy normalized,

$$
\int U_{E_{\mathrm{col}} L}(R) U_{E_{\mathrm{col}}^{\prime} L}(R) d R=\delta\left(E-E^{\prime}\right)
$$

The radial wave functions are found by numerically solving

$$
\left\{-\frac{d^{2}}{d R^{2}}+\frac{L(L+1)}{R^{2}}+2 \mu\left[V_{0}(R)-E_{\mathrm{col}}\right]\right\} U_{E_{\mathrm{col},}, L}(R)=0
$$

subject to boundary conditions

$$
\begin{aligned}
& U_{E_{\mathrm{col}}, L}(0)=0, \\
& U_{E_{\mathrm{col}}, L}(R) \simeq \sqrt{\frac{\mu k R^{2}}{2 \pi \hbar^{2}}}\left[h_{L}^{(2)}(k R)+S_{L}(k) h_{L}^{(1)}(k R)\right] .
\end{aligned}
$$

In the above, $V_{0}(R)$ is the isotropic interaction, $\mu$ is the reduced mass, $E_{\mathrm{col}}$ is the collision energy, $k=\sqrt{2 \mu E_{\mathrm{col}}}$ is the wave number, $L$ is the partial wave quantum number, and $h_{L}^{(1)}$ and $h_{L}^{(2)}$ denote spherical Hankel functions of the first and second kind, respectively. ${ }^{21}$

Using the Wigner-Eckart theorem, the required matrix element of the dipole moment surface is

$$
\begin{aligned}
\left\langle\left(N_{A} N_{B}\right) N L ; J M E_{\mathrm{col}}\left|\hat{\mu}_{v}\right|\left(N_{A}^{\prime} N_{B}^{\prime}\right) N^{\prime} L^{\prime} ; J^{\prime} M^{\prime} E_{\mathrm{col}}^{\prime}\right\rangle & \\
= & (-1)^{J-M}\left(\begin{array}{ccc}
J & 1 & J^{\prime} \\
-M & v & M^{\prime}
\end{array}\right) \\
& \times\left\langle\left(N_{A} N_{B}\right) N L ; J E_{\mathrm{col}}\|\hat{\mu}\|\left(N_{A}^{\prime} N_{B}^{\prime}\right) N^{\prime} L^{\prime} ; J^{\prime} E_{\mathrm{col}}^{\prime}\right\rangle,
\end{aligned}
$$

where $\mu_{0}=\mu_{z}$ and $\mu_{ \pm 1}=\mp\left(\mu_{x} \pm i \mu_{y}\right) / \sqrt{2}$ are the spherical components of the dipole operator. The symbol in round braces is a Wigner 3-jm symbol, and the reduced matrix element of the dipole operator is given by

$$
\begin{aligned}
& \left\langle\left(N_{A} N_{B}\right) N L ; J E_{\mathrm{col}}\|\hat{\mu}\|\left(N_{A}^{\prime} N_{B}^{\prime}\right) N^{\prime} L^{\prime} ; J^{\prime} E_{\mathrm{col}}^{\prime}\right\rangle \\
& =\sum_{l_{1}, l_{2}, l, \lambda}\left\langle E_{\mathrm{col}}, L\left|D_{l_{1}, l_{2}, l, \lambda}(R)\right| E_{\mathrm{col}}^{\prime}, L^{\prime}\right\rangle \\
& \quad \times\left\langle\left(N_{A} N_{B}\right) N L ; J \|\left[\left[C^{\left(l_{1}\right)}\left(\hat{r}_{A}\right) \otimes C^{\left(l_{2}\right)}\left(\hat{r}_{B}\right)\right]^{(l)} \otimes C^{(\lambda)}(\hat{R})\right]^{(1)}\right. \\
& \left.\quad \times \|\left(N_{A}^{\prime} N_{B}^{\prime}\right) N^{\prime} L^{\prime} ; J^{\prime}\right\rangle
\end{aligned}
$$

and Eq. (A9). The symbol in rectangular brackets denotes the Clebsch-Gordan coupled spherical harmonics in the Racah normalization, in which the dipole moment is expanded, and $D_{l_{1}, l_{2}, l, \lambda}(R)$ are the corresponding expansion coefficients. See the Appendix for more details. The complete $M, M^{\prime}$, and $v$ dependence of the spectral density thus amounts to the square of a 3-jm symbol, and the sum over these quantum numbers yields

$$
\sum_{M, M^{\prime}, v}\left(\begin{array}{ccc}
J & 1 & J^{\prime} \\
-M & v & M^{\prime}
\end{array}\right)^{2}=\left\{J, 1, J^{\prime}\right\},
$$

where the triangular delta $\left\{j_{1}, j_{2}, j_{3}\right\}$ equals unity if the triad satisfies the triangular conditions $\left|j_{1}-j_{2}\right| \leq j_{3} \leq j_{1}+j_{2}$ and zero otherwise. ${ }^{22}$ Thus, the spectral density becomes

$$
\begin{aligned}
V g(\omega, T)= & \lambda_{0}^{3} \hbar \sum_{N_{A}, N_{B}, N} \sum_{N_{A}^{\prime}, N_{B}^{\prime}, N^{\prime}} P_{N_{A}} P_{N_{B}} \\
& \times \sum_{L, L^{\prime}} \sum_{J, J^{\prime}} \int_{0}^{\infty} d E_{\mathrm{col}} \exp \left(-\frac{E_{\mathrm{col}}}{k_{B} T}\right) \\
& \times \mid\left\langle\left(N_{A} N_{B}\right) N L ; J E_{c o l}\|\hat{\mu}\|\left(N_{A}^{\prime} N_{B}^{\prime}\right) N^{\prime} L^{\prime} ;\left.J^{\prime} E_{\mathrm{col}}^{\prime}\right|^{2},\right.
\end{aligned}
$$


where the final-state kinetic energy, $E_{\text {col }}^{\prime}$, is related to the initial-state kinetic energy, $E_{\mathrm{col}}$, and the rotational quanta by the requirement of energy conservation, imposed by the integral over the delta function in Eq. (8).
In order to perform the sums over $J, J^{\prime}, N$, and $N^{\prime}$, we first expand the square in Eq. (15) as a double sum over the components of the dipole surface, $\left\{l_{1}, l_{2}, l, \lambda\right\}$, yielding for the spectral density

$$
\begin{aligned}
& V g(\omega, T)=\lambda_{0}^{3} \hbar \sum_{N_{A}, N_{B}, N} \sum_{N_{A}^{\prime}, N_{B}^{\prime}, N^{\prime}} P_{N_{A}} P_{N_{B}} \sum_{L, L^{\prime}} \sum_{J, J^{\prime}} \sum_{l_{1}, l_{2}, l, \lambda} \sum_{l_{1}^{\prime}, l_{2}^{\prime}, l^{\prime}, \lambda^{\prime}} \\
& \times \int_{0}^{\infty} d E_{\mathrm{col}} \exp \left(-\frac{E_{\mathrm{col}}}{k_{B} T}\right)\left\langle E_{\mathrm{col}}, L\left|D_{l_{1}, l_{2}, l, \lambda}\right| E_{\mathrm{col}}^{\prime}, L^{\prime}\right\rangle\left\langle E_{\mathrm{col}}, L\left|D_{l_{1^{\prime}}, l_{2}^{\prime}, l^{\prime}, \lambda^{\prime}}\right| E_{\mathrm{col}}^{\prime}, L^{\prime}\right\rangle \\
& \times\left[l, l^{\prime}\right]^{1 / 2}\left[1, J, J^{\prime}, L, L^{\prime}, N, N^{\prime}, N_{A}, N_{A}^{\prime}, N_{B}, N_{B}^{\prime}\right]\left(\begin{array}{ccc}
L & \lambda & L^{\prime} \\
0 & 0 & 0
\end{array}\right)\left(\begin{array}{ccc}
L & \lambda^{\prime} & L^{\prime} \\
0 & 0 & 0
\end{array}\right) \\
& \times\left(\begin{array}{ccc}
N_{A} & l_{1} & N_{A}^{\prime} \\
0 & 0 & 0
\end{array}\right)\left(\begin{array}{ccc}
N_{A} & l_{1}^{\prime} & N_{A}^{\prime} \\
0 & 0 & 0
\end{array}\right)\left(\begin{array}{ccc}
N_{B} & l_{2} & N_{B}^{\prime} \\
0 & 0 & 0
\end{array}\right)\left(\begin{array}{ccc}
N_{B} & l_{2}^{\prime} & N_{B}^{\prime} \\
0 & 0 & 0
\end{array}\right) \\
& \times\left\{\begin{array}{ccc}
N_{A} & N_{A}^{\prime} & l_{1} \\
N_{B} & N_{B}^{\prime} & l_{2} \\
N & N^{\prime} & l
\end{array}\right\}\left\{\begin{array}{ccc}
N_{A} & N_{A}^{\prime} & l_{1}^{\prime} \\
N_{B} & N_{B}^{\prime} & l_{2}^{\prime} \\
N & N^{\prime} & l^{\prime}
\end{array}\right\}\left\{\begin{array}{ccc}
N & N^{\prime} & l \\
L & L^{\prime} & \lambda \\
J & J^{\prime} & 1
\end{array}\right\}\left\{\begin{array}{ccc}
N & N^{\prime} & l^{\prime} \\
L & L^{\prime} & \lambda^{\prime} \\
J & J^{\prime} & 1
\end{array}\right\},
\end{aligned}
$$

where the symbols in curly braces are Wigner 9- $j$ symbols and we use the short-hand notation

$$
\left[j_{1}, j_{2}, \ldots, j_{n}\right] \equiv\left(2 j_{1}+1\right)\left(2 j_{2}+1\right) \cdots\left(2 j_{n}+1\right) .
$$

Thus, the complete $J$ and $J^{\prime}$ dependence is contained in a product of 9- $j$ symbols and dimension factors, and the sum over these quantum numbers can be performed using the orthogonality relation ${ }^{22}$

$$
\begin{gathered}
\sum_{X, Y}[X, Y]\left\{\begin{array}{ccc}
j_{1} & j_{2} & k \\
j_{3} & j_{4} & l \\
X & Y & j_{5}
\end{array}\right\}\left\{\begin{array}{ccc}
j_{1} & j_{2} & k^{\prime} \\
j_{3} & j_{4} & l^{\prime} \\
X & Y & j_{5}
\end{array}\right\} \\
=\frac{\delta_{k k^{\prime}} \delta_{l l^{\prime}}}{[l, k]}\left\{j_{1} j_{2} k\right\}\left\{j_{3} j_{4} l\right\}\left\{k l j_{5}\right\} .
\end{gathered}
$$

Next, the complete $N$ and $N^{\prime}$ dependence is contained in a similar sum. Evaluating this sum also using the orthogonality relation, Eq. (18), yields

$$
\begin{aligned}
V g(\omega, T)= & \lambda_{0}^{3} \hbar \sum_{l_{1}, l_{2}, l, \lambda} \sum_{N_{A}, N_{B}, N_{A}^{\prime}, N_{B}^{\prime}} P_{N_{A}} P_{N_{B}} \\
& \times \sum_{L, L^{\prime}} \frac{\left[1, L, L^{\prime}, N_{A}, N_{A}^{\prime}, N_{B}, N_{B}^{\prime}\right]}{\left[l_{1}, l_{2}, \lambda\right]} \\
& \times\left(\begin{array}{ccc}
N_{A} & l_{1} & N_{A}^{\prime} \\
0 & 0 & 0
\end{array}\right)^{2}\left(\begin{array}{ccc}
N_{B} & l_{2} & N_{B}^{\prime} \\
0 & 0 & 0
\end{array}\right)^{2} \\
& \times\left(\begin{array}{ccc}
L & \lambda & L^{\prime} \\
0 & 0 & 0
\end{array}\right)^{2} \int_{0}^{\infty} \exp \left(-\frac{E_{\mathrm{col}}}{k_{B} T}\right) \\
& \times\left|\left\langle E_{\mathrm{col}}, L\left|D_{l_{1}, l_{2}, l, \lambda}\right| E_{\mathrm{col}}^{\prime}, L^{\prime}\right\rangle\right|^{2} d E_{\mathrm{col}} .
\end{aligned}
$$

The remaining sums over $N_{A}, N_{B}, N_{A}^{\prime}$, and $N_{B}^{\prime}$ cannot be performed analytically. In the isotropic interaction approximation, the spectrum consists of an incoherent sum over the components $\left\{l_{1}, l_{2}, l, \lambda\right\}$ of the dipole moment. The spectral density can also be cast into the following insightful form:

$$
\begin{aligned}
V g(\omega)= & \sum_{l_{1}, l_{2}, l, \lambda} \sum_{N_{A}, N_{B}, N_{A}^{\prime}, N_{B}^{\prime}} P_{N_{A}} P_{N_{B}} \frac{\left[1, N_{A}, N_{A}^{\prime}, N_{B}, N_{B}^{\prime}\right]}{\left[l_{1}, l_{2}, \lambda\right]} \\
& \times\left(\begin{array}{ccc}
N_{A} & l_{1} & N_{A}^{\prime} \\
0 & 0 & 0
\end{array}\right)^{2}\left(\begin{array}{ccc}
N_{B} & l_{2} & N_{B}^{\prime} \\
0 & 0 & 0
\end{array}\right)^{2} \\
& \times V G_{l_{1}, l_{2}, l, \lambda}\left(\omega-\omega_{\mathrm{rot}}\right)
\end{aligned}
$$

with

$$
\begin{aligned}
V G_{l_{1}, l_{2}, l, \lambda}(\omega)= & \hbar \lambda_{0}^{3} \sum_{L, L^{\prime}}\left[L, L^{\prime}\right]\left(\begin{array}{ccc}
L & \lambda & L^{\prime} \\
0 & 0 & 0
\end{array}\right)^{2} \\
& \times \int_{0}^{\infty} d E_{\mathrm{col}} \exp \left(-\frac{E_{\mathrm{col}}}{k_{B} T}\right) \\
& \times\left|\left\langle E_{\mathrm{col}}, L\left|D_{l_{1}, l_{2}, l, \lambda}\right| E_{\mathrm{col}}+\hbar \omega, L^{\prime}\right\rangle\right|^{2} .
\end{aligned}
$$

This shows that the spectrum can be thought of as a superposition of a basic translational profile, $V G(\omega)$, centered at the rotational transitions given by $\hbar \omega_{\text {rot }}=E_{\text {rot }}^{\prime}-E_{\text {rot }}{ }^{4}$

We note that the above results are equivalent to those obtained in Ref. 4. When comparing, one should note the difference in the definition of the expansion of the dipole moment surface due to the use of Racah normalized spherical harmonics.

\section{Isotropic interactions between indistinguishable molecules}

If the colliding molecules are indistinguishable, the statistical weights also depend on the exchange symmetry of the scattering wave function. Therefore, one should consider scattering wave functions which are adapted to permutation 
symmetry. This can be done as follows:

$$
\begin{aligned}
\left|\left(N_{A} N_{B}\right) N L ; J M \pm\right\rangle \\
=\left[2\left(1+\delta_{N_{A} N_{B}}\right)\right]^{-1 / 2}\left(1 \pm \hat{P}_{A B}\right)\left|\left(N_{A} N_{B}\right) N L ; J M\right\rangle \\
=\left[2\left(1+\delta_{N_{A} N_{B}}\right)\right]^{-1 / 2}\left[\left|\left(N_{A} N_{B}\right) N L ; J M\right\rangle\right. \\
\left.\quad \pm(-1)^{N_{A}+N_{B}-N+L}\left|\left(N_{B} N_{A}\right) N L ; J M\right\rangle\right]
\end{aligned}
$$

where $N_{B} \geq N_{A}$ in order to ensure linear independence of the wave functions. Next, we rederive an expression for the spectral density, using the following symmetry adapted functions as initial and final states:

$\left|\left(N_{A} N_{B}\right) N L ; J M E_{\mathrm{col}} \pm\right\rangle=\left|\left(N_{A} N_{B}\right) N L ; J M \pm\right\rangle\left|E_{\mathrm{col}}, L\right\rangle$.

Inserting these states into Eq. (2), the analog of Eq. (8) becomes

$$
\begin{aligned}
V g(\omega, T)= & \hbar \sum_{N_{A}, N_{B}, N, \epsilon} \sum_{N_{A}^{\prime}, N_{B}^{\prime}, N^{\prime}} \sum_{L, L^{\prime}} \int_{0}^{\infty} \int_{0}^{\infty} d E_{\mathrm{col}} d E_{\mathrm{col}}^{\prime} P_{N_{A} N_{B}}^{\epsilon}\left(E_{\mathrm{col}}, T\right) \\
& \times \sum_{J, J, M, M,, \nu}\left|\left\langle\left(N_{A} N_{B}\right) N L ; J M E_{\mathrm{col}} \epsilon\left|\hat{\mu}_{\nu}\right|\left(N_{A}^{\prime} N_{B}^{\prime}\right) N^{\prime} L^{\prime} ; J^{\prime} M^{\prime} E_{\mathrm{col}}^{\prime} \epsilon\right\rangle\right|^{2} \delta\left(E_{\mathrm{tot}}^{\prime}-E_{\mathrm{tot}}-\hbar \omega\right) .
\end{aligned}
$$

The Boltzmann weight depends on $N_{A}$ and $N_{B}$, as well as on the permutation symmetry, $\epsilon= \pm 1$, and is given by

$$
P_{N_{A} N_{B}}^{\epsilon}\left(E_{\mathrm{col}}, T\right)=\frac{\lambda_{0}^{3}}{V Z_{\mathrm{rot}}} g_{N_{A} N_{B}}^{\epsilon} \exp \left(-\frac{E_{\mathrm{col}}+E_{\mathrm{rot}}}{k_{B} T}\right) .
$$

The statistical weights, denoted $g_{N_{A} N_{B}}^{\epsilon}$, are discussed in Sec. III $B$ and their values for all symmetry types of $\mathrm{H}_{2}-\mathrm{H}_{2}$ are given in Table I.

Now, the spectral density contains matrix elements of the form

$$
\begin{aligned}
&\left\langle\left(N_{A} N_{B}\right) N L ; J M E_{\mathrm{col}} \pm\left|\hat{\mu}_{v}\right|\left(N_{A}^{\prime} N_{B}^{\prime}\right) N^{\prime} L^{\prime} ; J^{\prime} M^{\prime} E_{\mathrm{col}}^{\prime} \pm\right\rangle \\
&= {\left[\left(1+\delta_{N_{A} N_{B}}\right)\left(1+\delta_{N_{A}^{\prime} N_{B}^{\prime}}\right)\right]^{-1 / 2}\left\langle\left(N_{A} N_{B}\right) N L ; J M E_{\mathrm{col}}\right| } \\
& \times \hat{\mu}_{v}\left(1 \pm \hat{P}_{A B}\right)\left|\left(N_{A}^{\prime} N_{B}^{\prime}\right) N^{\prime} L^{\prime} ; J^{\prime} M^{\prime} E_{\mathrm{col}}^{\prime}\right\rangle
\end{aligned}
$$

where use has been made of the fact that $\hat{P}_{A B}$ commutes with $\hat{\mu}_{v}$ and that $\left(1 \pm \hat{P}_{A B}\right)^{2}=2\left(1 \pm \hat{P}_{A B}\right)$. Since this matrix element is the sum of two terms, its square is expanded as four terms. The sums over $J, J^{\prime}, M, M^{\prime}$, and $v$ can be performed as in Sec. II B. The same holds for the sums over $N$ and $N^{\prime}$ for the two direct terms, but for the two exchange terms, one encounters sums of the form ${ }^{22}$

$$
\begin{gathered}
\sum_{N, N^{\prime}}(-1)^{N^{\prime}}\left[N, N^{\prime}\right]\left\{\begin{array}{ccc}
N_{A} & N_{A}^{\prime} & l_{1} \\
N_{B} & N_{B}^{\prime} & l_{2} \\
N & N^{\prime} & l
\end{array}\right\}\left\{\begin{array}{ccc}
N_{A} & N_{B}^{\prime} & l_{1}^{\prime} \\
N_{B} & N_{A}^{\prime} & l_{2}^{\prime} \\
N & N^{\prime} & l
\end{array}\right\} \\
=(-1)^{2 N_{B}+l_{2}+l_{2}^{\prime}}\left\{\begin{array}{ccc}
N_{A} & N_{A}^{\prime} & l_{1} \\
N_{B}^{\prime} & N_{B} & l_{2} \\
l_{1}^{\prime} & l_{2}^{\prime} & l
\end{array}\right\} .
\end{gathered}
$$

Evaluating these sums, the spectral density becomes

$$
\begin{aligned}
& V g(\omega)=\lambda_{0}^{3} \hbar \sum_{l_{1}, l_{2}, l_{1}^{\prime}, l_{2}^{\prime}, l, \lambda} \sum_{N_{A}, N_{B}, N_{A}^{\prime}, N_{B}^{\prime}, \epsilon, L^{\prime}} g_{N_{A} N_{B}}^{\epsilon} Z_{\mathrm{rot}}^{-1} \exp \left(-\frac{E_{\mathrm{rot}}}{k_{B} T}\right) \int d E_{\mathrm{col}} \exp \left(-\frac{E_{\mathrm{col}}}{k_{B} T}\right) \\
& \times \frac{\left[1, L, L^{\prime}, N_{A}, N_{A}^{\prime}, N_{B}, N_{B}^{\prime}\right]}{\left(1+\delta_{N_{A} N_{B}}\right)\left(1+\delta_{N_{A}^{\prime} N_{B}^{\prime}}\right)[\lambda]}\left\langle E_{\mathrm{col}}, L\left|D_{l_{1}, l_{2}, l, \lambda}\right| E_{\mathrm{col}}^{\prime}, L^{\prime}\right\rangle\left\langle E_{\mathrm{col}}, L\left|D_{l_{1}^{\prime}, l_{2}^{\prime}, l, \lambda}\right| E_{\mathrm{col}}^{\prime}, L^{\prime}\right\rangle\left(\begin{array}{ccc}
L & \lambda & L^{\prime} \\
0 & 0 & 0
\end{array}\right)^{2} \\
& \times\left[\frac{\delta_{l_{1} l_{1}} \delta_{l_{2} l_{2}^{\prime}}}{\left[l_{1}, l_{2}\right]}\left[\left(\begin{array}{ccc}
N_{A} & l_{1} & N_{A}^{\prime} \\
0 & 0 & 0
\end{array}\right)^{2}\left(\begin{array}{ccc}
N_{B} & l_{2} & N_{B}^{\prime} \\
0 & 0 & 0
\end{array}\right)^{2}+\left(\begin{array}{ccc}
N_{A} & l_{1} & N_{B}^{\prime} \\
0 & 0 & 0
\end{array}\right)^{2}\left(\begin{array}{ccc}
N_{B} & l_{2} & N_{A}^{\prime} \\
0 & 0 & 0
\end{array}\right)^{2}\right]\right. \\
& \left.+2 \epsilon(-1)^{N_{A}^{\prime}+N_{B}^{\prime}+L^{\prime}+l_{2}+l_{2}^{\prime}}\left(\begin{array}{ccc}
N_{A} & l_{1} & N_{A}^{\prime} \\
0 & 0 & 0
\end{array}\right)\left(\begin{array}{ccc}
N_{A} & l_{1}^{\prime} & N_{B}^{\prime} \\
0 & 0 & 0
\end{array}\right)\left(\begin{array}{ccc}
N_{B} & l_{2} & N_{B}^{\prime} \\
0 & 0 & 0
\end{array}\right)\left(\begin{array}{ccc}
N_{B} & l_{2}^{\prime} & N_{A}^{\prime} \\
0 & 0 & 0
\end{array}\right)\left\{\begin{array}{ccc}
N_{A} & N_{A}^{\prime} & l_{1} \\
N_{B}^{\prime} & N_{B} & l_{2} \\
l_{1}^{\prime} & l_{2}^{\prime} & l
\end{array}\right)\right\} \text {, }
\end{aligned}
$$

where use has been made of the fact that the 9- $j$ symbols are invariant under reflection in their diagonal, and that one can interchange the summation indices $l_{1}$ and $l_{1}^{\prime}$ and $l_{2}$ and $l_{2}^{\prime}$.
In contrast to the distinguishable particle case where the spectral density is an incoherent sum over the dipole components, $\left\{l_{1}, l_{2}, l, \lambda\right\}$, interference occurs between terms with 
TABLE I. Nuclear spin statistical weights for all symmetries. The rotational quanta of both monomers are denoted $N_{A}$ and $N_{B}$, respectively, and $\epsilon$ denotes the symmetry under permutation of the monomers.

\begin{tabular}{lrrc}
\hline \hline$(-1)^{N_{A}}$ & $(-1)^{N_{B}}$ & $\epsilon$ & $g_{N_{A} N_{B}}^{\epsilon}$ \\
\hline 1 & 1 & 1 & 1 \\
1 & 1 & -1 & 0 \\
-1 & -1 & 1 & 6 \\
-1 & -1 & -1 & 3 \\
\pm 1 & $\mp 1$ & & 3 \\
\hline \hline
\end{tabular}

$l_{1} \neq l_{1}^{\prime}$ and $l_{2} \neq l_{2}^{\prime}$ and the spectral lines are now labeled with the six indices, $\left\{l_{1}, l_{1}^{\prime}, l_{2}, l_{2}^{\prime}, l, \lambda\right\}$. However, if symmetric and antisymmetric states are given equal weights in the thermal average, their contributions to the interference cancel exactly, and the spectral density is zero unless $l_{1}=l_{1}^{\prime}$ and $l_{2}=l_{2}^{\prime}$. In this case, the resulting spectrum is identical to the one obtained by the distinguishable particle formalism described above. The spectrum will deviate if symmetric and antisymmetric states are not given equal weights, for example, due to the nuclear spin statistics of indistinguishable particles.

We note that our discussion differs from previous treatment of exchange symmetry. ${ }^{4}$ Previously, wave functions were not explicitly adapted to exchange symmetry, rather a statistical weighing of different partial waves was applied. This works well in the limit of low temperatures, where all molecules are in the rotational ground state, $N_{A}=N_{B}=0$, such that $N=0$ and the permutation symmetry is determined by $(-1)^{L}$ alone.

Even at the lowest temperatures that we consider $(T=77 \mathrm{~K})$, the $N_{X}=1$ rotational states are populated. Therefore, we cannot apply the method of Ref. 4, in which exchange is only taken into account through statistical weights of different partial waves. Furthermore, we note that a partial wave dependent weight only alters the translational profile, which contributes equally to each rotational line. Thus, the theory of Ref. 4 cannot predict the relative effect of exchange on the different rotational transitions. For these reasons, we use the more rigorous treatment described above, explicitly adapting spatial wave functions to exchange symmetry.

\section{Algorithm for treating anisotropic interactions}

For anisotropic interaction potentials, one can no longer separate the translational and rotational degrees of freedom. That is, the wave function can no longer be written as in Eq. (4). One can, however, introduce an angular basis, $\left\{\left|\phi_{i}\right\rangle\right\}$, see, for example, Eq. (5), and expand the total wave function in this channel basis, as

$$
\left\langle R \mid \Psi_{q}\right\rangle=\frac{1}{R} \sum_{p}\left|\phi_{p}\right\rangle U_{p q}(R) .
$$

The expansion coefficients, which depend only on the radial coordinate, can be obtained with coupled-channels theory. ${ }^{23}$ That is, the wave function, Eq. (29), can be inserted a timeindependent Schrödinger equation, which can be solved numerically, subject to scattering boundary conditions. In this section, we derive a method to calculate the dipole coupling between coupled-channels wave functions on-the-fly, that is, without storing the many-component wave function on the radial grid points.

Inserting the above expansion of the wave function into the time independent Schrödinger equation

$$
\begin{gathered}
(\hat{H}-E)\left|\Psi_{q}\right\rangle=0, \\
\hat{H}=-\frac{\hbar^{2}}{2 \mu R} \frac{d^{2}}{d R^{2}} R+\frac{\hat{L}^{2}}{2 \mu R^{2}}+\hat{H}_{A}+\hat{H}_{B}+\hat{V}
\end{gathered}
$$

and taking scalar products with the channel functions yields the following differential equation for the expansion coefficients:

$$
\frac{d^{2}}{d R^{2}} \mathbf{U}=\mathbf{W} \mathbf{U}
$$

Here, the W-matrix is Hermitian, and its elements are given by

$$
W_{p^{\prime} p}=2 \mu\left\langle\phi_{p^{\prime}}\left|\frac{\hat{L}^{2}}{2 \mu R^{2}}+\hat{H}_{A}+\hat{H}_{B}+\hat{V}-E\right| \phi_{p}\right\rangle .
$$

The coupled-channels equations are to be solved subject to the scattering boundary conditions. For energy-normalized scattering states, the boundary conditions are

$$
\begin{aligned}
U_{p q}(0) & =0, \\
U_{p q}(R) & \simeq \sqrt{\frac{\mu k_{p} R^{2}}{2 \pi \hbar^{2}}}\left[h_{L_{p}}^{(2)}\left(k_{p} R\right) \delta_{p q}+h_{L_{p}}^{(1)}\left(k_{p} R\right) S_{p q}\right],
\end{aligned}
$$

with $h_{L_{p}}^{(n)}(x)$ the spherical Hankel functions and $L_{p}$ the partial wave quantum number for channel $p$. Here, it has been assumed that the channel functions diagonalize the asymptotic Hamiltonian $\hat{H}_{A}+\hat{H}_{B}$. Their eigenvalues, the channel energies $\epsilon_{p}$, determine the wave numbers $k_{p}=\hbar^{-1} \sqrt{2 \mu\left(E-\epsilon_{p}\right)}$. If the asymptotic Hamiltonian is not diagonal in the channel basis, a transformation to the asymptotic basis, which diagonalizes the asymptotic Hamiltonian, is required.

Direct propagation of the solutions $U_{p q}(R)$ is numerically unstable due to the fact that they vary exponentially in classically forbidden regions. There exists a number of algorithms which avoid such instabilities, of which renormalized and logderivative propagation are the most popular. ${ }^{24}$ For the purpose of describing collision-induced absorption, we require the complete wave function and not just the asymptotic form. Therefore, it is most convenient to use renormalized propagation, ${ }^{24}$ as the calculated Q-matrices allow to reconstruct the full wave function straight-forwardly, by successive multiplication of the asymptotic wave function. However, we note that explicitly calculating many-component wave functions for a large number of radial grid points requires a lot of computer memory. Therefore, we derive a method for the direct propagation of the required integrals.

First, we divide the angular basis in two distinct sets, one $\left\{\left|\phi_{p}\right\rangle \mid p=1,2, \ldots, r\right\}$ is associated with the initial states and one $\left\{\left|\phi_{p}\right\rangle \mid p=r+1, r+2, \ldots, r+s\right\}$ with the final states. Note that such a distinction can always be made, since initial and final states coupled by the dipole operator have opposite parity, and parity is conserved in a collision process. Thus, the matrix of expansion coefficients takes the block form 


$$
\mathbf{U}_{j}=\left[\begin{array}{c|c}
\tilde{\mathbf{U}}_{j}^{(i)} & \mathbf{0} \\
\hline \mathbf{0} & \tilde{\mathbf{U}}_{j}^{(f)}
\end{array}\right],
$$

where the subscripts denote evaluation at some point of the discrete grid in the radial coordinate $\left\{R_{j} \mid j=1,2, \ldots, n\right\}$. Here, we shall assume this grid to be equidistant with grid spacing $\Delta_{R}$.

In renormalized propagation, we define the Q-matrix through

$$
\mathbf{U}_{j}=\mathbf{Q}_{j+1} \mathbf{U}_{j+1} .
$$

The short-range boundary conditions in Eq. (33) yield the first Q-matrix, $\mathbf{Q}_{1}=\mathbf{0}$. The Q-matrix is then propagated outwards using recursion relations that may be derived from Eq. (31), augmented with some approximation. For example, the renormalized Numerov method uses a Taylor expansion of the wave function up to fifth order in the grid spacing $\Delta_{R} \cdot{ }^{24}$ When the angular basis is partitioned in the two sets defined above, the Q-matrices also take the block form

$$
\mathbf{Q}_{j}=\left[\begin{array}{c|c}
\tilde{\mathbf{Q}}_{j}^{(i)} & \mathbf{0} \\
\hline \mathbf{0} & \tilde{\mathbf{Q}}_{j}^{(f)}
\end{array}\right],
$$

where the blocks satisfy

$$
\tilde{\mathbf{U}}_{j}^{(x)}=\tilde{\mathbf{Q}}_{j+1}^{(x)} \tilde{\mathbf{U}}_{j+1}^{(x)},
$$

and the blocks can be propagated separately for $x=i, f$.

Now, we wish to calculate a matrix element of the dipole moment, which, when evaluated in $R=R_{j}$, is represented in the angular basis by the matrix

$$
\mathbf{D}_{j}=\left[\begin{array}{c|c}
\mathbf{0} & \tilde{\mathbf{D}}_{j} \\
\hline \tilde{\mathbf{D}}_{j}^{\dagger} & \mathbf{0}
\end{array}\right] .
$$

The matrix element of the dipole operator in Eq. (2) can be expressed, using a mid-point rule quadrature in $R$, as

$$
\mathbf{A}_{n}=\Delta_{R} \sum_{j=1}^{n} \tilde{\mathbf{U}}_{j}^{(i) \dagger} \tilde{\mathbf{D}}_{j} \tilde{\mathbf{U}}_{j}^{(f)} .
$$

It is convenient to express this integral in terms of the wave functions at the end of the interval, according to

$$
\begin{aligned}
\mathbf{A}_{n}= & \tilde{\mathbf{U}}_{n}^{(i) \dagger} \mathbf{B}_{n} \tilde{\mathbf{U}}_{n}^{(f)}, \\
\mathbf{B}_{n}= & \Delta_{R} \sum_{j=1}^{n-1}\left(\tilde{\mathbf{Q}}_{j+1}^{(i)} \tilde{\mathbf{Q}}_{j+2}^{(i)} \ldots \tilde{\mathbf{Q}}_{n}^{(i)}\right)^{\dagger} \\
& \times \tilde{\mathbf{D}}_{j}\left(\tilde{\mathbf{Q}}_{j+1}^{(f)} \tilde{\mathbf{Q}}_{j+2}^{(f)} \ldots \tilde{\mathbf{Q}}_{n}^{(f)}\right)+\Delta_{R} \tilde{\mathbf{D}}_{n} .
\end{aligned}
$$

To derive a recursion relation, we consider

$$
\begin{aligned}
\mathbf{B}_{n+1}= & \Delta_{R} \sum_{j=1}^{n}\left(\tilde{\mathbf{Q}}_{j+1}^{(i)} \tilde{\mathbf{Q}}_{j+2}^{(i)} \ldots \tilde{\mathbf{Q}}_{n+1}^{(i)}\right)^{\dagger} \\
& \times \tilde{\mathbf{D}}_{j}\left(\tilde{\mathbf{Q}}_{j+1}^{(f)} \tilde{\mathbf{Q}}_{j+2}^{(f)} \ldots \tilde{\mathbf{Q}}_{n+1}^{(f)}\right)+\Delta_{R} \tilde{\mathbf{D}}_{n+1} \\
= & \Delta_{R} \tilde{\mathbf{Q}}_{n+1}^{(i) \dagger}\left[\sum_{j=1}^{n-1}\left(\tilde{\mathbf{Q}}_{j+1}^{(i)} \tilde{\mathbf{Q}}_{j+2}^{(i)} \ldots \tilde{\mathbf{Q}}_{n}^{(i)}\right)^{\dagger}\right. \\
& \left.\times \tilde{\mathbf{D}}_{j}\left(\tilde{\mathbf{Q}}_{j+1}^{(f)} \tilde{\mathbf{Q}}_{j+2}^{(f)} \ldots \tilde{\mathbf{Q}}_{n}^{(f)}\right)+\tilde{\mathbf{D}}_{n}\right] \tilde{\mathbf{Q}}_{n+1}^{(f)}+\Delta_{R} \tilde{\mathbf{D}}_{n+1} \\
= & \tilde{\mathbf{Q}}_{n+1}^{(i) \dagger} \mathbf{B}_{n} \tilde{\mathbf{Q}}_{n+1}^{(f)}+\Delta_{R} \tilde{\mathbf{D}}_{n+1} .
\end{aligned}
$$

This allows one to directly propagate the dipole coupling and match to the asymptotic form of $\widetilde{\mathbf{U}}^{(x)}$, Eq. (33), for $x=i, f$ at the last grid point using Eq. (40). The three matrices $\mathbf{Q}^{(i)}, \mathbf{Q}^{(f)}$, and $\mathbf{B}$ are propagated outwards simultaneously, but without coupled treatment of the initial and final states.

The method described above allows to propagate the dipole coupling between scattering states. With this dipole coupling, we can calculate the spectral density as

$$
\begin{aligned}
V g(\omega) & =\hbar V \int d E_{\mathrm{tot}} \sum_{i, f} P^{(i)} \sum_{M, M^{\prime}, v}\left|\left\langle i\left|\hat{\mu}_{\nu}\right| f\right\rangle\right|^{2} \\
& =\hbar \frac{\lambda_{0}^{3}}{Z_{\mathrm{rot}}} \int d E_{\mathrm{tot}} \exp \left(-\frac{E_{\mathrm{tot}}}{k_{B} T}\right) \sum_{i, f} g_{N_{A} N_{B}}^{\epsilon}|\langle i\|\hat{\mu}\| f\rangle|^{2} .
\end{aligned}
$$

Here, the sum over $i$ runs over all scattering states at total energy $E_{\text {tot }}$. The sum over $f$ is over all scattering states at total energy $E_{\text {tot }}+\hbar \omega$. The permutation symmetry of the final state may be restricted to that of the initial state. The parity of the final state is opposite to that of the initial state, and the finalstate total angular momentum differs from that of the initial state by at most one unit, $|J-1| \leq J^{\prime} \leq J+1$. Note that the sum over the projection quantum numbers has already been carried out using Eq. (14). The required integral, $\langle i\|\hat{\mu}\| f\rangle$, is computed using the method described above, on discrete grids in $E$ and $\omega$. For indistinguishable molecules, the statistical weights, $g_{N_{A} N_{B}}^{\epsilon}$, depend on the permutation symmetry of the initial state, $\epsilon$, and the scattering states should be adapted to this symmetry in order to account for exchange effects. The statistical weights are discussed in more detail in Sec. III B, and the values for all symmetry types of the $\mathrm{H}_{2}-\mathrm{H}_{2}$ system are given in Table I.

We note that the method described above can also be used to calculate collision-induced absorption spectra in certain approximation schemes, in addition to providing the numerically exact solution from coupled-channels calculations. For example, one could use this method in combination with an isotropic potential to obtain the result of the isotropic interaction theory. To save computational time, one should then exploit the fact that such an approximation scheme block diagonalizes the W-matrix, Eq. (32), and propagate the blocks separately. We illustrate this idea in the companion paper, where we calculate collision-induced absorption spectra of $\mathrm{N}_{2}-\mathrm{N}_{2}$ within the coupled-states approximation. ${ }^{13}$

\section{APPLICATION TO $\mathrm{H}_{2}-\mathrm{H}_{2}$}

\section{A. Potential energy and dipole moment surface}

The description of the collision-induced absorption of $\mathrm{H}_{2}$ pairs requires accurate potential energy and dipole moment surfaces, i.e., the potential energy and electric dipole moment as a function of the geometry of the collision complex. These are taken from Ref. 25 . In this section, we briefly explain how these surfaces were obtained, and the analytical form to which they were fit.

The potential energy was calculated for the $\mathrm{H}_{2}-\mathrm{H}_{2}$ complex, using the spin-restricted coupled-clusters method with 
single and double excitations with perturbative triples $[\operatorname{CCSD}(\mathrm{T})]$. At the same level of theory, the dipole moment was determined from the linear response to a finite electric field. Two field strengths were used to ensure that hyperpolarizability effects are suppressed. The calculations were performed in an augmented correlation consistent quadruple zeta one-electron basis set (aug-cc-pVQZ). All energies were corrected for the basis set super position error. ${ }^{26}$ The calculations were performed for 18 orientations and 17 separations of the two molecules. The $\mathrm{H}_{2}$ bond length was kept fixed at its vibrational average of $1.449 a_{0}$.

The potential energy and dipole moment surfaces were fit, in a linear least squares procedure, to an expansion in coupled spherical harmonics. In the spherical harmonics expansion of the potential, all terms with $l_{1}+l_{2} \leq 6$ were included. This introduces a root mean square error of less than $0.2 \%$ for any separation. For the dipole moment, the expansion was truncated including only terms with $l_{1}+l_{2} \leq 4$. At short separation, the error introduced by this truncation increases to $2 \%$ at $R=3.7 a_{0}$ but should be smaller than $1 \%$ at separations that contribute significantly to the absorption.

In order to evaluate these expansions for arbitrary intermolecular distances, $R$, we performed a fit of the expansion coefficients $V_{l_{1}, l_{2}, l}(R)$ and $D_{l_{1}, l_{2}, l, \lambda}(R)$. First, the long-range behavior was fit to an expansion in $1 / R$, using a $R^{n}$ weighted linear least squares fit, with $n$ determined by the leading power of $1 / R$. For the potential energy, we fit terms with $n \leq 7$ for $R \geq 12 a_{0}$, whereas for the dipole surface, only the $n=4$ terms $\left\{l_{1}, l_{2}, l, \lambda\right\}=\{2,0,2,3\}$ and $\{2,2,2,3\}$ were fit. The long-range contributions were damped by multiplication with Tang-Toennies damping functions, ${ }^{27}$

$$
f_{n, \beta}(R)=1-e^{-\beta R} \sum_{k=0}^{n} \frac{(\beta R)^{k}}{k !} .
$$

We used $\beta=1.48 a_{0}^{-1}$ for the potential energy surface and $\beta$ $=1.62 a_{0}^{-1}$ for the dipole moment surface. These damped longrange contributions were subtracted from the total to obtain the short-range part, which was fit for $R \leq 12 a_{0}$ using the reproducing kernel Hilbert space method. ${ }^{28}$ The smoothness parameter for this fit was set to two, and the asymptotic behavior was chosen such that the short-range part falls off as the first term in the asymptotic expansion that was not fit explicitly in the long-range.

\section{B. Nuclear spin statistics}

In this work, we consider all hydrogen nuclei to be protons, which is the naturally most abundant hydrogen isotope. This nucleus is a fermion with nuclear spin quantum number $I=1 / 2$, such that the total nuclear spin of molecule $X=A, B$ takes the values $I_{X}=0,1$. Since the total wave function should be antisymmetric with respect to the exchange of identical protons, the antisymmetric singlet $\left(I_{X}=0\right)$ spin states combine with symmetric rotational states (even $N_{X}$ ), and the symmetric triplet $\left(I_{X}=1\right)$ spin states combine with antisymmetric rotational states (odd $N_{X}$ ). If we consider the two molecules to be distinguishable, the statistical weight for the rotational states of the complex is given by the product $g_{N_{A}} g_{N_{B}}$ with
$g_{N_{X}}=1$ if $N_{X}$ is even and $g_{N_{X}}=3$ if $N_{X}$ is odd. In case we consider all feasible permutations of nuclei, given by the permutation of both nuclei in each molecule, and the permutation of the whole molecules, the statistical weights also depend on the permutation symmetry, $\epsilon$. The group theoretical calculation of the spin statistical weights is treated in the book of Bunker and Jensen..$^{29}$ Here, we merely state the result in Table I.

\section{Details of calculations with isotropic potentials}

We define the functions

$$
\begin{aligned}
\tilde{f}_{l_{1}, l_{2}, l_{1}^{\prime}, l_{2}^{\prime}, l, \lambda}^{+}\left(\omega_{\text {trans }}, E_{\mathrm{col}}\right)= & \sum_{\text {even } L, \text { odd } L^{\prime}}\left[L, L^{\prime}\right]\left(\begin{array}{lll}
L & \lambda & L^{\prime} \\
0 & 0 & 0
\end{array}\right)^{2} \\
& \times\left\langle E_{\mathrm{col}}, L\left|D_{l_{1} l_{2} l \lambda}\right| E_{\mathrm{col}}^{\prime}, L^{\prime}\right\rangle \\
& \times\left\langle E_{\mathrm{col}}, L\left|D_{l_{1}^{\prime} l_{2}^{\prime} l \lambda \mid}\right| E_{\mathrm{col}}^{\prime}, L^{\prime}\right\rangle, \\
\tilde{f}_{l_{1}, l_{2}, l_{1}^{\prime}, l_{2}^{\prime}, l, \lambda}^{-}\left(\omega_{\text {trans }}, E_{\mathrm{col}}\right)= & \sum_{\text {odd } L, \text { even } L^{\prime}}\left[L, L^{\prime}\right]\left(\begin{array}{lll}
L & \lambda & L^{\prime} \\
0 & 0 & 0
\end{array}\right)^{2} \\
& \times\left\langle E_{\mathrm{col}}, L\left|D_{l_{1} l_{2} l \lambda}\right| E_{\mathrm{col}}^{\prime}, L^{\prime}\right\rangle \\
& \times\left\langle E_{\mathrm{col}}, L\left|D_{l_{1}^{\prime} l_{2}^{\prime} l \lambda \mid}\right| E_{\mathrm{col}}^{\prime}, L^{\prime}\right\rangle .
\end{aligned}
$$

These functions are computed on discrete logarithmic grids in the kinetic energies, $E_{\mathrm{col}}$ and $E_{\mathrm{col}}^{\prime}$, ranging from $0.1 \mathrm{~K}$ to $3000 \mathrm{~K}$ in 100 steps. For $l_{1}=l_{1}^{\prime}$ and $l_{2}=l_{2}^{\prime}$, the functions $\tilde{f}_{l_{1}, l_{2}, l_{1}, l_{2}, l, \lambda}\left(\omega_{\text {trans }}, E_{\mathrm{col}}\right)$ are evaluated on a discrete grid of frequencies that satisfy $\hbar \omega_{\text {trans }}=E_{\mathrm{col}}^{\prime}-E_{\mathrm{col}}$, rather than on a grid in $E_{\text {col }}^{\prime}$, by spline interpolation of $\log \left(\tilde{f}_{l_{1}, l_{2}, l_{1}, l_{2}, l, \lambda}^{ \pm}\right)$, followed by exponentiation. These functions can be used to compute the following integrals for arbitrary temperature:

$$
\begin{aligned}
f_{l_{1}, l_{2}, l_{1}^{\prime}, l_{2}^{\prime}, l, \lambda}^{ \pm}\left(\omega_{\text {trans }}, T\right)= & \hbar \lambda_{0}^{3} \int_{0}^{\infty} d E_{\text {col }} \exp \left(-\frac{E_{\text {col }}}{k_{B} T}\right) \\
& \times \tilde{f}_{l_{1}, l_{2}, l_{1}^{\prime}, l_{2}^{\prime}, l, \lambda}\left(\omega_{\text {trans }}, E_{\text {col }}\right) .
\end{aligned}
$$

Numerically, these integrals were evaluated using trapezoidal integration. The spectral density is computed as

$$
\begin{aligned}
V g(\omega, T)= & \sum_{l_{1}, l_{2}, l, \lambda} \sum_{N_{A}, N_{B}} P_{N_{A}} P_{N_{B}} \\
& \times \sum_{N_{A}^{\prime}, N_{B}^{\prime}}\left(\begin{array}{ccc}
N_{A} & l_{1} & N_{A}^{\prime} \\
0 & 0 & 0
\end{array}\right)^{2}\left(\begin{array}{ccc}
N_{B} & l_{2} & N_{B}^{\prime} \\
0 & 0 & 0
\end{array}\right)^{2} \\
& \times \frac{\left[1, N_{A}, N_{A}^{\prime}, N_{B}, N_{B}^{\prime}\right]}{\left[l_{1}, l_{2}, \lambda\right]} \\
& \times\left[f_{l_{1}, l_{2}, l_{1}, l_{2}, l, \lambda}^{+}\left(\omega-\omega_{\text {rot }}, T\right)\right. \\
& \left.+f_{l_{1}, l_{2}, l_{1}, l_{2}, l, \lambda}^{-}\left(\omega-\omega_{\text {rot }}, T\right)\right]
\end{aligned}
$$

with $\hbar \omega_{\text {rot }}=E_{\text {rot }}^{\prime}-E_{\text {rot }}$.

For the calculations adapted to exchange symmetry, the spectral density is calculated as 


$$
\begin{aligned}
& V g(\omega, T)=\frac{1}{Z_{\mathrm{rot}}} \sum_{l_{1}, l_{2}, l_{1}^{\prime}, l_{2}^{\prime}, l, \lambda} \sum_{N_{A}, N_{B}, N_{A}^{\prime}, N_{B}^{\prime}} \exp \left(-\frac{E_{\mathrm{rot}}}{k_{B} T}\right) \frac{\left[1, N_{A}, N_{A}^{\prime}, N_{B}, N_{B}^{\prime}\right]}{\left(1+\delta_{N_{A} N_{B}}\right)\left(1+\delta_{N_{A}^{\prime} N_{B}^{\prime}}\right)\left[l_{1}, l_{2}, \lambda\right]} \\
& \times\left\{\delta_{l_{1} l_{1}^{\prime}} \delta_{l_{2} l_{2}^{\prime}}\left[\left(\begin{array}{ccc}
N_{A} & l_{1} & N_{A}^{\prime} \\
0 & 0 & 0
\end{array}\right)^{2}\left(\begin{array}{ccc}
N_{B} & l_{2} & N_{B}^{\prime} \\
0 & 0 & 0
\end{array}\right)^{2}+\left(\begin{array}{ccc}
N_{A} & l_{1} & N_{B}^{\prime} \\
0 & 0 & 0
\end{array}\right)^{2}\left(\begin{array}{ccc}
N_{B} & l_{2} & N_{A}^{\prime} \\
0 & 0 & 0
\end{array}\right)^{2}\right]\right. \\
& \times\left(g_{N_{A} N_{B}}^{+}+g_{N_{A} N_{B}}^{-}\right)\left[f_{l_{1}, l_{2}, l_{1}, l_{2}, l, \lambda}^{+}\left(\omega-\omega_{\text {rot }}, T\right)+f_{l_{1}, l_{2}, l_{1}, l_{2}, l, \lambda}^{-}\left(\omega-\omega_{\text {rot }}, T\right)\right]+2\left[l_{1}, l_{2}\right](-1)^{N_{A}^{\prime}+N_{B}^{\prime}} \\
& \times\left(\begin{array}{ccc}
N_{A} & l_{1} & N_{A}^{\prime} \\
0 & 0 & 0
\end{array}\right)\left(\begin{array}{ccc}
N_{A} & l_{1}^{\prime} & N_{B}^{\prime} \\
0 & 0 & 0
\end{array}\right)\left(\begin{array}{ccc}
N_{B} & l_{2} & N_{B}^{\prime} \\
0 & 0 & 0
\end{array}\right)\left(\begin{array}{ccc}
N_{B} & l_{2}^{\prime} & N_{A}^{\prime} \\
0 & 0 & 0
\end{array}\right)\left\{\begin{array}{ccc}
N_{A} & N_{A}^{\prime} & l_{1} \\
N_{B}^{\prime} & N_{B} & l_{2} \\
l_{1}^{\prime} & l_{2}^{\prime} & l
\end{array}\right\} \\
& \left.\times\left(g_{N_{A} N_{B}}^{+}-g_{N_{A} N_{B}}^{-}\right)\left[f_{l_{1}, l_{2}, l_{1}^{\prime}, l_{2}^{\prime}, l, \lambda}^{-}\left(\omega-\omega_{\mathrm{rot}}, T\right)-f_{l_{1}, l_{2}, l_{1}^{\prime}, l_{2}^{\prime}, l, \lambda}^{+}\left(\omega-\omega_{\mathrm{rot}}, T\right)\right]\right\},
\end{aligned}
$$

with $\hbar \omega_{\text {rot }}=E_{\text {rot }}^{\prime}-E_{\text {rot }}$. In case $l_{1} \neq l_{1}^{\prime}$ or $l_{2} \neq l_{2}^{\prime}$, we use direct rather than logarithmic interpolation of $f_{l_{1}, l_{2}, l_{1}^{\prime}, l^{\prime}, l, \lambda}\left(\omega_{\text {trans }}, T\right)$. We recall that, in the case of the exchange adapted theory, the sums are constrained such that $N_{B} \geq N_{A}$, to ensure linear independence of the scattering states.

In all cases, whether adapted to exchange symmetry or not, the absorption coefficient is calculated from the spectral density, as discussed in Sec. II A, according to

$$
\alpha(\omega, T)=\frac{2 \pi^{2}}{3 \hbar c} n^{2} \omega\left[1-\exp \left(-\frac{\hbar \omega}{k_{B} T}\right)\right] V g(\omega, T) .
$$

The computationally most expensive part is the calculation of the radial integrals $\left\langle E_{\mathrm{col}}, L\left|D_{l_{1} l_{2} l \lambda}\right| E_{\mathrm{col}}^{\prime}, L^{\prime}\right\rangle$. The required wave functions are computed on a discrete equidistant grid in radial coordinate $R$, using the renormalized Numerov method. ${ }^{24}$ The calculated functions $\tilde{f}_{l_{1}, l_{2}, l_{1}^{\prime}, l_{2}^{\prime}, l, \lambda}\left(E, \omega_{\text {trans }}\right)$ were converged using a radial grid ranging from 4 to $100 a_{0}$, in steps of $0.1 a_{0}$. This step size corresponds to the shortest local de Broglie wave length, $\lambda_{\mathrm{db}}$, divided by 10 . The angular momentum quantum numbers $L$ and $N_{A}, N_{B}$ were truncated at $L_{\max }$ and $N_{\max }$, respectively. For the final state, we included all angular functions coupled to the initial state by the dipole moment surface of Ref. 25. We used $L_{\max }=30$ and $N_{\max }=20$, after checking that this leads to results which are converged better than to $1 \%$ accuracy.

\section{Details of calculations with anisotropic potentials}

The coupled-channels equations have been solved using the renormalized Numerov method, ${ }^{24}$ propagating the dipole coupling as described in Sec. II D. The grid in frequency was chosen equidistant with spacing $25 \mathrm{~cm}^{-1}$ up to $\omega=375 \mathrm{~cm}^{-1}$ and additional points at $600,800,1900,2000,2100$, and $2200 \mathrm{~cm}^{-1}$. The energy grid included 281 points, from 0.1 to $5000 \mathrm{~K}$. The energy grid was obtained as a logarithmically spaced grid, with additional points near the combined rotational energy levels of both molecules, to accurately sample scattering resonances and the opening of the rotationally excited channels. We use the angular basis functions of Eq. (A3), which are adapted to both inversion and permutation symmetry. This basis is truncated using the criterion that $N_{A}\left(N_{A}+1\right)+N_{B}\left(N_{B}+1\right) \leq N_{\max }\left(N_{\max }+1\right)$, with $N_{\max }=8$.
The radial grid ranged from 4 to $50 a_{0}$, again using a step size of $\lambda_{\mathrm{db}} / 10$. The total angular momentum was truncated at $J_{\max }=50$ for the highest energies, and lower otherwise. The thermal average in Eq. (42) has been calculated using a trapezoidal integration rule.

To save computational time, asymptotically closed channels, which are required only in the short-range, were removed in the long-range. To determine whether or not a channel should be excluded, its channel energy is compared to an exponentially decaying energy criterion, which was chosen such that all channels are included for $R<10 a_{0}$ and all asymptotically closed channels are removed for $R>20 a_{0}$. This idea has been exploited previously by Martinazzo et al. ${ }^{30}$

\section{E. Results}

In this section, the numerical results of the theory discussed above are presented and compared to experimental results by Birnbaum. ${ }^{2}$ In Fig. 1, the absorption spectrum is shown for three temperatures, $T=77,195$, and $292 \mathrm{~K}$. The measurements are represented by the dots, whereas lines represent theory in the isotropic interaction approximation. Crosses represent calculations including the interaction anisotropy,

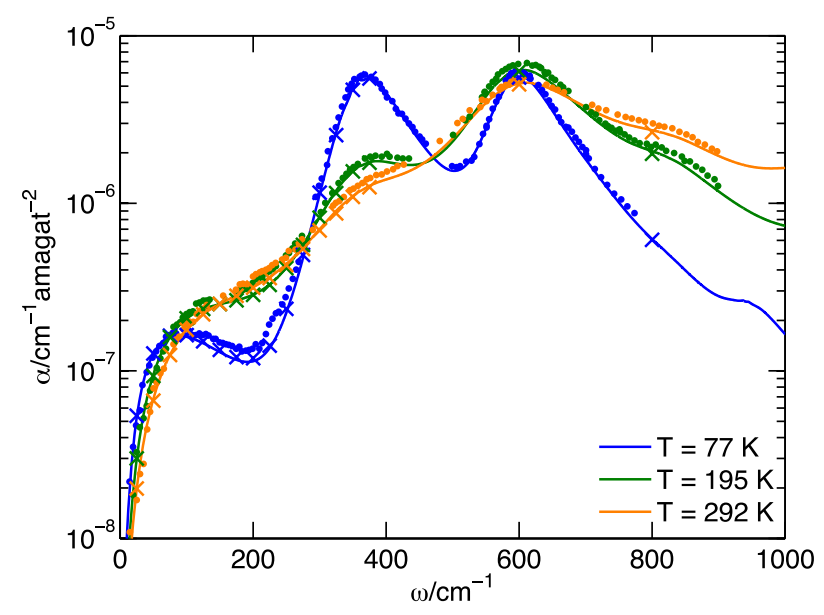

FIG. 1. Collision-induced absorption spectrum of $\mathrm{H}_{2}$ for different temperatures. Lines mark theory with an isotropic interaction potential and distinguishable particles, crosses mark theory with the full anisotropic potential, and the dots represent measurements in Ref. 2. 


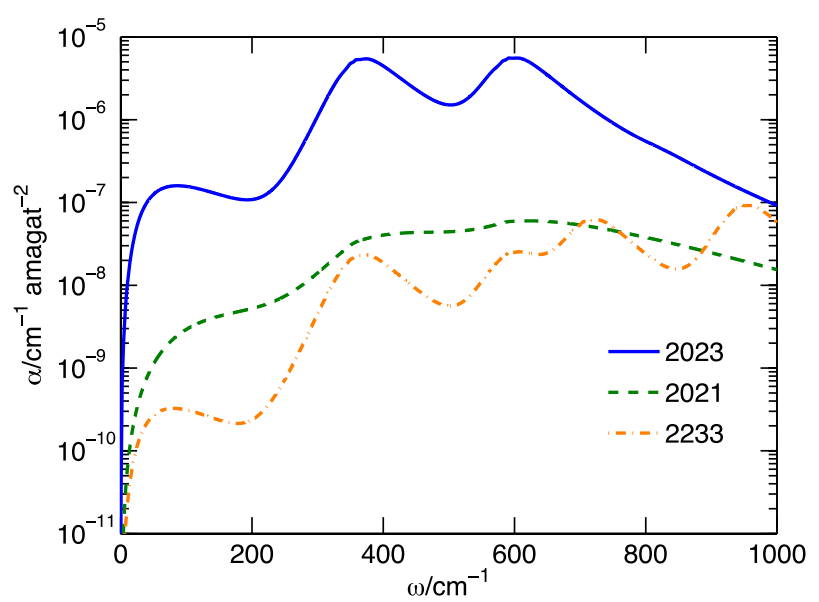

FIG. 2. Most important incoherent contributions $l_{1}, l_{2}, l, \lambda$, to the absorption spectrum at a temperature of $77 \mathrm{~K}$.

which are discussed below. Reasonable agreement between experiment and the isotropic interaction approximation theory is obtained. The difference between experiment and theory at the band maxima is around $8 \%$ for the lowest temperature. It is not completely clear what the accuracy of the experiment is, but differences between measurements by different authors are on the order of $\pm 10 \%$. $^{2,31-36}$

Figure 2 shows the different incoherent contributions to the spectrum at $T=77 \mathrm{~K}$. The spectrum is dominated by the $l_{1}, l_{2}, l, \lambda=2,0,2,3$ and $0,2,2,3$ terms. In first order perturbation theory, these terms correspond to the quadrupole moment of one molecule inducing a dipole moment in the complex through the isotropic polarizability of the other molucule. ${ }^{38}$ Higher spherical components contribute significantly at higher frequency, as they induce transitions with larger changes in the rotational quanta.

Figure 3 shows the most significant translational profiles for a temperature of $T=77 \mathrm{~K}$, as a function of the angular frequency associated with the difference in kinetic energy, $\hbar \omega_{\text {trans }}=E_{\text {col }}^{\prime}-E_{\text {col }}$. The translational profile can be used to explain the shape of the total spectrum. As discussed in Sec. II B, the spectrum is built up as a sum of translational

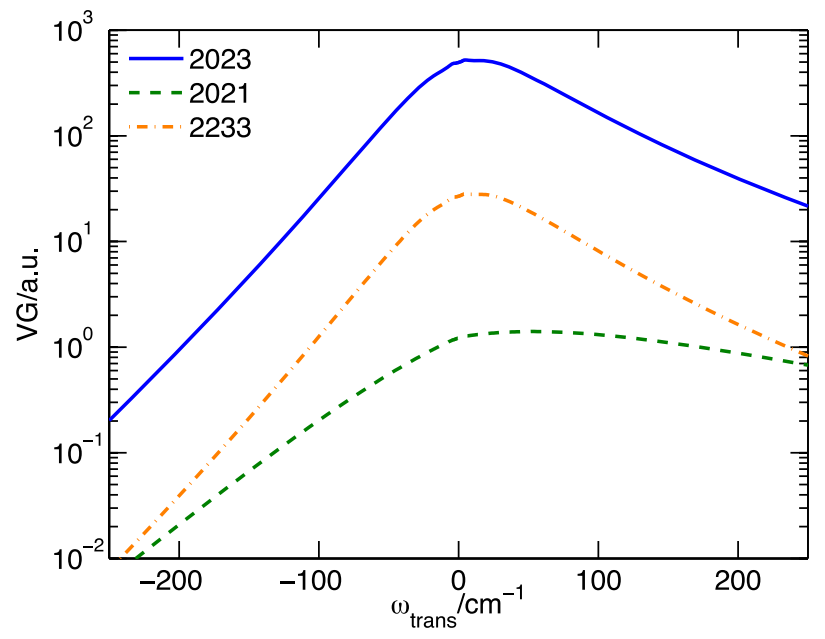

FIG. 3. Translational profile for some of the dominant incoherent contributions $l_{1}, l_{2}, l, \lambda$, at a temperature of $77 \mathrm{~K}$. profiles, centered around individual rotational transitions. The width of these profiles leads to a coarsely structured spectrum, with broad but clearly visible absorption peaks near the $N_{X}$ $=0 \rightarrow 2\left(\omega=354 \mathrm{~cm}^{-1}\right)$ and $N_{X}=1 \rightarrow 3\left(\omega=587 \mathrm{~cm}^{-1}\right)$ rotational transitions. Dipole coupling between states with identical rotational energy, for example, $N_{X}=1 \rightarrow 1$, contributes to a translational band at low photon frequency. With increasing temperature, the translational profiles broaden, and the intensity shifts to higher $\omega$, due to the increased population of higher rotational levels.

We note that the peak intensity of a rotational line is found at slightly higher frequency than the actual rotational transition. This follows from the fact that the translational profiles peak at some $\omega_{\max }>0$, which can be explained in the following way. The odd parity of the dipole operator requires the partial wave to change in an absorption process. This means that the initial and final states feel a slightly different effective potential, due to the different centrifugal barrier. Therefore, maximum overlap of translational wave functions does not occur for zero energy difference, as it would if the effective potentials were identical, but for some small but non-zero energy difference. Furthermore, the translational profile is not symmetric around $\omega=0$. In fact, the red wing is weaker than the blue wing by a frequency dependent Boltzmann factor, $V G(-\omega)=\exp \left(-\hbar \omega / k_{B} T\right) V G(\omega)$, since the red and the blue wing are related by detailed balance. ${ }^{19}$ Hence, the maximum occurs for $\omega_{\max }>0$, and the rotational lines in the spectrum appear to be shifted to the blue.

Figure 4 shows the theoretical absorption spectrum at $T=77 \mathrm{~K}$, including exchange effects, computed with the permutation adapted formalism derived in Sec. II C. The effects amount to about $2 \%$ on the $N_{X}=0 \rightarrow 2$ transition. This is in agreement with Ref. 4 , in which work exchange effects were explored with an approximate partial-wave-weighing formalism. This confirms their general conclusion that exchange effects are insignificant even at temperatures as low as $T=77 \mathrm{~K}$. The peculiar shape of the exchange effects originates from the difference in position of the maxima of the translational profiles for even and odd partial waves. The difference of the two profiles, which determines the exchange effects, is therefore sharply peaked. The double transition

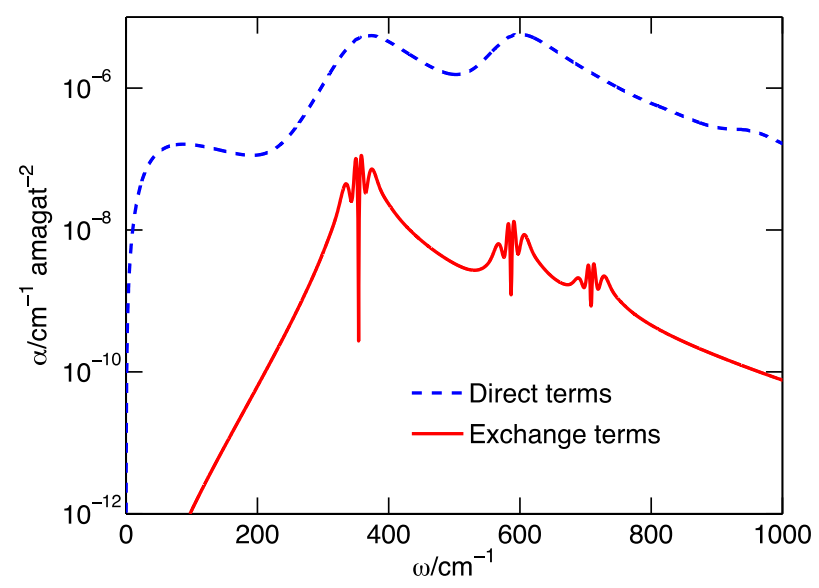

FIG. 4. Collision-induced absorption spectrum of $\mathrm{H}_{2}$ at $T=77 \mathrm{~K}$, including exchange effects. 


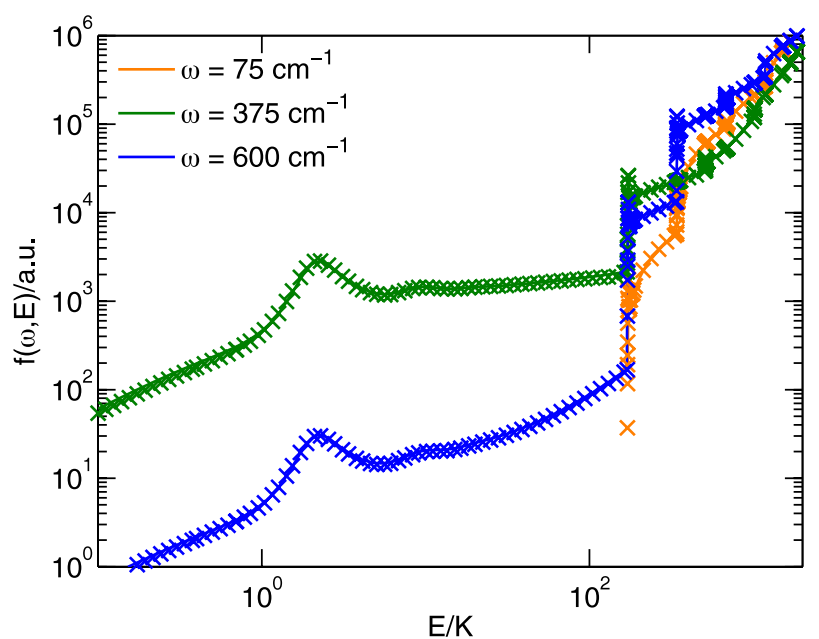

FIG. 5. Line strength as a function of initial-state energy. The lines represent the isotropic interaction approximation calculation, and the crosses represent the anisotropic calculation. Exchange effects are suppressed.

$N_{A}=0 \rightarrow 2, N_{B}=0 \rightarrow 2$ at $\omega=708 \mathrm{~cm}^{-1}$ is clearly visible in the exchange terms, whereas it is not discernible in the direct spectrum. By contrast, the double transition $N_{A}=0$ $\rightarrow 2, N_{B}=1 \rightarrow 3$ at $\omega=941 \mathrm{~cm}^{-1}$ is visible in the absorption spectrum, but there is no exchange contribution as this transition involves one ortho- $\mathrm{H}_{2}$ and one para $-\mathrm{H}_{2}$ molecule.

The theoretical absorption spectrum, obtained using the full anisotropic potential, is shown by the crosses in Fig. 1. The results for isotropic and anisotropic calculations agree closely, due to large rotational constant of $\mathrm{H}_{2}$, which effectively renders the hydrogen molecule isotropic.

Figure 5 shows the line strength as a function of the initialstate energy. The crosses represent the anisotropic calculation, where the line strength is given by

$$
f\left(\omega, E_{\mathrm{tot}}\right)=\sum_{i, f} g_{N_{A} N_{B}}^{\epsilon}|\langle i\|\hat{\mu}\| f\rangle|^{2} .
$$

In this equation, the sum over $i$ is restricted to all states at energy $E_{\mathrm{tot}}$, whereas the sum over $f$ is restricted to states of energy $E_{\mathrm{tot}}+\hbar \omega$. The solid line represents results of the isotropic interaction approximation. Neglecting exchange effects, the line strength is calculated as

$$
\begin{aligned}
& f(\omega, E)=\sum_{l_{1}, l_{2}, l, \lambda} \sum_{N_{A}, N_{B}, N_{A}^{\prime}, N_{B}^{\prime}} g_{N_{A}} g_{N_{B}} \\
& \times \sum_{L, L^{\prime}} \frac{\left[1, L, L^{\prime}, N_{A}, N_{A}^{\prime}, N_{B}, N_{B}^{\prime}\right]}{\left[l_{1}, l_{2}, \lambda\right]} \\
& \times\left(\begin{array}{ccc}
N_{A} & l_{1} & N_{A}^{\prime} \\
0 & 0 & 0
\end{array}\right)^{2}\left(\begin{array}{ccc}
N_{B} & l_{2} & N_{B}^{\prime} \\
0 & 0 & 0
\end{array}\right)^{2}\left(\begin{array}{ccc}
L & \lambda & L^{\prime} \\
0 & 0 & 0
\end{array}\right)^{2} \\
& \times\left|\left\langle E-E_{\mathrm{rot}}, L\left|D_{l_{1}, l_{2}, l, \lambda}\right| E-E_{\mathrm{rot}}^{\prime}+\hbar \omega, L^{\prime}\right\rangle\right|^{2} \text {. }
\end{aligned}
$$

The agreement of these two results further illustrates the validity of the isotropic interaction approximation for $\mathrm{H}_{2}-\mathrm{H}_{2}$. Figure 5 shows that agreement of the spectra calculated using isotropic or anisotropic potentials does not follow from a fortunate cancellation of errors in the integral over the initial-state energy.

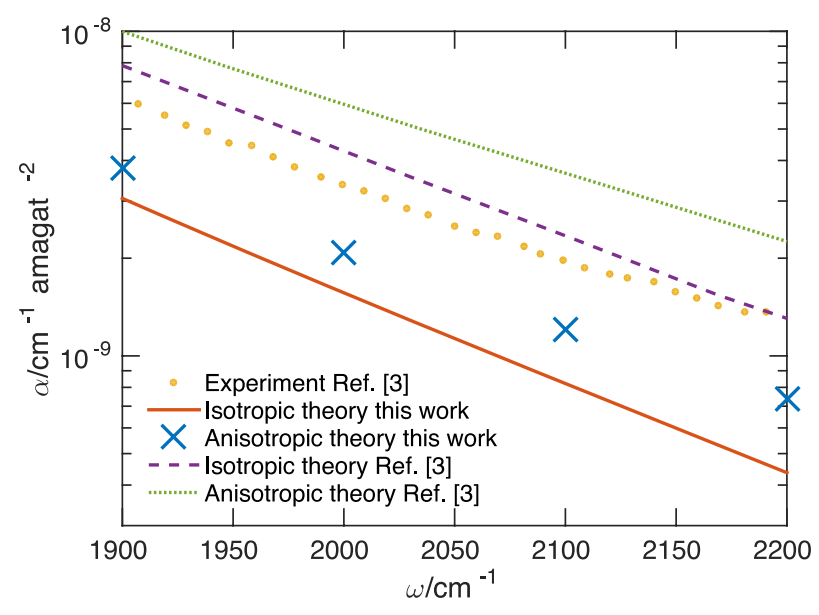

FIG. 6. Far wing of the collision-induced absorption spectrum of normal $\mathrm{H}_{2}$ (3:1 ratio of ortho:para hydrogen) at $T=77 \mathrm{~K}$.

Finally, we consider the far wing of the collision-induced absorption spectrum, for frequencies around $\omega=2000 \mathrm{~cm}^{-1}$. Measurements in this spectral region have been presented in Ref. 3, along with first principles calculations using the full anisotropic potential of Ref. 37. We have performed similar calculations using the more recent potential energy and dipole moment surfaces of Ref. 25, calculating the dipole coupling on-the-fly, as described in this work. In Fig. 6, we compare our results to the experimental and theoretical results of Ref. 3. For both potentials, the effect of anisotropic interactions is to increase the absorption significantly, by a factor of 2 in the calculations of Ref. 3 and by $70 \%$ in the present work, both at the highest frequencies. The line shapes obtained in both calculations are very similar, and they match the experimental line shape reasonably well. The overall intensity is not well reproduced, however, as the previous calculations with anisotropic potentials overestimate the absorption by $60 \%$, and our results underestimate the intensity by about $40 \%$. As pointed out in Ref. 3, the far wing of the spectrum is sensitive to the repulsive part of the potential, as well as to higher spherical components of the dipole moment. These are more difficult to calculate accurately and do not affect the lower frequency part of the spectrum, hence it is possible that the potential energy and dipole moment surfaces are still insufficiently accurate to reproduce the far wing absorption.

\section{CONCLUSIONS}

We have discussed three formalisms to describe the collision-induced absorption of $\mathrm{H}_{2}$ molecules. All calculations are completely from first principles, using the accurate potential energy and dipole moment surfaces of Ref. 25. First, we discuss the established theory of collision-induced absorption assuming an isotropic interaction between two distinguishable molecules. Then, we rederive the theory, rigorously adapting the wave functions to exchange symmetry. Finally, anisotropic interactions are taken into account in a novel method. Our method is computationally very efficient since we treat the absorption perturbatively, with direct propagation of the dipole transition matrix. 
Good agreement with experiment is found when using the simplest model, assuming isotropic interactions between distinguishable molecules. Exchange effects, within the isotropic interaction approximation, are seen to amount to only a few percent at the lowest temperatures studied. The effect of interaction anisotropy on the collision-induced absorption of molecular hydrogen is significant only in the far wing of the spectrum. In the companion paper, ${ }^{13}$ we study the effect of interaction anisotropy on the collision-induced absorption of the $\mathrm{N}_{2}-\mathrm{N}_{2}$ system, which is more profound.

\section{ACKNOWLEDGMENTS}

The authors gratefully acknowledge the computer and communication department (CNCZ) of the science faculty of the Radboud University Nijmegen for the computer resources made available and for technical support.

\section{APPENDIX: ANGULAR FUNCTIONS AND MATRIX ELEMENTS}

The angular dependence of the scattering wave function can be expanded in products of angular momentum kets, $\left|N_{A} M_{A}\right\rangle\left|N_{B} M_{B}\right\rangle\left|L M_{L}\right\rangle$, describing the rotation of molecules $A$ and $B$ and the end-over-end rotation of the system. It is convenient to construct eigenfunctions of the total angular momentum as

$$
\begin{aligned}
& \left|\left(N_{A} N_{B}\right) N L ; J M\right\rangle \\
& \quad=\sum_{\substack{M_{A}, M_{B}, M_{N}, M_{L} \\
\quad}}\left|N_{A} M_{A}\right\rangle\left|N_{B} M_{B}\right\rangle\left|L M_{L}\right\rangle \\
& \quad\left\langle N_{A} M_{B} M_{B} \mid N M_{N}\right\rangle\left\langle N M_{N} L M_{L} \mid J M\right\rangle .
\end{aligned}
$$

The total angular momentum, $J$, and its space-fixed projection, $M$, are conserved quantities. These states are also eigenfunctions of the spatial inversion operator, $\hat{i}$, as well as of $\hat{P}_{12}^{(A)}$ and $\hat{P}_{12}^{(B)}$, which interchange the two atoms of molecules $A$ and $B$, respectively,

$$
\begin{aligned}
\hat{i}\left|\left(N_{A} N_{B}\right) N L ; J M\right\rangle & =(-1)^{N_{A}+N_{B}+L}\left|\left(N_{A} N_{B}\right) N L ; J M\right\rangle, \\
\hat{P}_{12}^{(A)}\left|\left(N_{A} N_{B}\right) N L ; J M\right\rangle & =(-1)^{N_{A}}\left|\left(N_{A} N_{B}\right) N L ; J M\right\rangle, \quad \text { (A2) } \\
\hat{P}_{12}^{(B)}\left|\left(N_{A} N_{B}\right) N L ; J M\right\rangle & =(-1)^{N_{B}}\left|\left(N_{A} N_{B}\right) N L ; J M\right\rangle .
\end{aligned}
$$

If molecules $A$ and $B$ are identical, permutation symmetry is also conserved, and these functions can be adapted to this symmetry according to

$$
\begin{aligned}
& \left|\left(N_{A} N_{B}\right) N L ; J M \pm\right\rangle \\
& =\frac{1 \pm \hat{P}_{A B}}{\sqrt{2\left(1+\delta_{N_{A} N_{B}}\right)}}\left|\left(N_{A} N_{B}\right) N L ; J M\right\rangle \\
& =\frac{1}{\sqrt{2\left(1+\delta_{N_{A} N_{B}}\right)}}\left[\left|\left(N_{A} N_{B}\right) N L ; J M\right\rangle\right. \\
& \left.\quad \pm(-1)^{N_{A}+N_{B}-N+L}\left|\left(N_{B} N_{A}\right) N L ; J M\right\rangle\right],
\end{aligned}
$$

where $\hat{P}_{A B}$ interchanges the two molecules.

In this appendix, we present matrix elements, required throughout this paper, in the angular basis Eq. (A1). In case one works with a $\hat{P}_{A B}$ adapted basis, one can relate the required matrix elements to the ones in the primitive basis using Eq. (A3) and the fact that $\hat{P}_{A B}$ is Hermitian, unitary, and commutes with both $\hat{H}$ and $\hat{\mu}$.

First, we note that the angular functions of Eq. (A1) are eigenstates of the asymptotic Hamiltonian

$$
\begin{aligned}
\hat{H}_{\text {asym }} & =\hat{H}_{A}+\hat{H}_{B}, \\
\hat{H}_{X} & =B_{0}^{(X)} \hat{N}_{X}^{2}-D_{0}^{(X)} \hat{N}_{X}^{4}, \\
\hat{H}_{X}\left|\left(N_{A} N_{B}\right) N L ; J M\right\rangle= & E_{\mathrm{rot}, N_{X}}\left|\left(N_{A} N_{B}\right) N L ; J M\right\rangle, \\
E_{\mathrm{rot}, N_{X}}= & B_{0}^{(X)} N_{X}\left(N_{X}+1\right) \\
& -D_{0}^{(X)}\left[N_{X}\left(N_{X}+1\right)\right]^{2},
\end{aligned}
$$

for $X=A, B$.

The potential energy surface is expanded in terms of coupled spherical harmonics, as

$$
\begin{aligned}
V\left(\hat{r}_{A}, \hat{r}_{B}, \vec{R}\right)= & \sum_{l_{1}, l_{2}, l} V_{l_{1}, l_{2}, l}(R) \\
& \times\left[\left[C^{\left(l_{1}\right)}\left(\hat{r}_{A}\right) \otimes C^{\left(l_{2}\right)}\left(\hat{r}_{B}\right)\right]^{(l)} \otimes C^{(l)}(\hat{R})\right]_{0}^{(0)} .
\end{aligned}
$$

The irreducible spherical tensor product is denoted

$$
\left[A^{\left(l_{1}\right)} \otimes B^{\left(l_{2}\right)}\right]_{m}^{(l)}=\sum_{m_{1}, m_{2}} A_{m_{1}}^{\left(l_{1}\right)} B_{m_{2}}^{\left(l_{2}\right)}\left\langle l_{1} m_{1} l_{2} m_{2} \mid l m\right\rangle,
$$

the spherical components of the tensors $C^{(l)}(\hat{R})$ are Racah normalized spherical harmonics depending on the polar angles of $\vec{R}$. For homonuclear diatomic molecules, only even $l_{1}$ and $l_{2}$ occur. Since parity is conserved, only even $l$ occurs. If $A$ and $B$ are identical molecules, one also has the symmetry $V_{l_{2}, l_{1}, l}(R)$ $=V_{l_{1}, l_{2}, l}(R)$. The potential energy surface can be determined from $a b$ initio electronic structure calculations, as discussed in Sec. III A for the case of $\mathrm{H}_{2}-\mathrm{H}_{2}$. The term with $l_{1}=l_{2}=l=0$ represents the isotropic part of the potential, whereas the other terms represent anisotropic components.

The dipole moment can also be expanded in terms of Clebsch-Gordan coupled spherical harmonics

$$
\begin{aligned}
\mu_{v}\left(\hat{r}_{A}, \hat{r}_{B}, \vec{R}\right)= & \sum_{l_{1}, l_{2}, l, \lambda} D_{l_{1}, l_{2}, l, \lambda}(R) \\
& \times\left[\left[C^{\left(l_{1}\right)}\left(\hat{r}_{A}\right) \otimes C^{\left(l_{2}\right)}\left(\hat{r}_{B}\right)\right]^{(l)} \otimes C^{(\lambda)}(\hat{R})\right]_{v}^{(1)} .
\end{aligned}
$$

Again, $l_{1}$ and $l_{2}$ are even due to the symmetry of homonuclear diatomic molecules. For the dipole moment, $l$ can differ from $\lambda$ by one, at most. Furthermore, only odd $\lambda$ occurs, due to the odd parity of the dipole operator. If $A$ and $B$ are identical molecules, the following symmetry holds: $D_{l_{2}, l_{1}, l, \lambda}(R)$ $=(-1)^{l+1} D_{l_{1}, l_{2}, l, \lambda}(R)$. The dipole moment surfaces can also be calculated $a b$ initio, as described in Sec. III A.

The required matrix elements of the angular terms in the expansion of the potential are given by 


$$
\begin{aligned}
\left\langle\left(N_{A} N_{B}\right) N L ; J\left|\left[\left[C^{\left(l_{1}\right)}\left(\hat{r}_{A}\right) \otimes C^{\left(l_{2}\right)}\left(\hat{r}_{B}\right)\right]^{(l)} \otimes C^{(l)}(\hat{R})\right]_{0}^{(0)}\right|\left(N_{A}^{\prime} N_{B}^{\prime}\right) N^{\prime} L^{\prime} ; J^{\prime}\right\rangle \\
=\delta_{J J^{\prime}}(-1)^{N_{A}+N_{B}+l+N^{\prime}+J}\left[N_{A}, N_{A}^{\prime}, N_{B}, N_{B}^{\prime}, N, N^{\prime}, L, L^{\prime}\right]^{1 / 2} \\
\quad \times\left(\begin{array}{ccc}
N_{A} & l_{1} & N_{A}^{\prime} \\
0 & 0 & 0
\end{array}\right)\left(\begin{array}{ccc}
N_{B} & l_{2} & N_{B}^{\prime} \\
0 & 0 & 0
\end{array}\right)\left(\begin{array}{ccc}
L & l & L^{\prime} \\
0 & 0 & 0
\end{array}\right)\left\{\begin{array}{ccc}
N & N^{\prime} & l \\
L^{\prime} & L & J
\end{array}\right\}\left\{\begin{array}{ccc}
N_{A} & N_{A}^{\prime} & l_{1} \\
N_{B} & N_{B}^{\prime} & l_{2} \\
N & N^{\prime} & l
\end{array}\right\} .
\end{aligned}
$$

The required reduced matrix elements of the angular terms in the expansion of the dipole are given by

$$
\begin{aligned}
& \left\langle\left(N_{A} N_{B}\right) N L ; J\left\|\left[\left[C^{\left(l_{1}\right)}\left(\hat{r}_{A}\right) \otimes C^{\left(l_{2}\right)}\left(\hat{r}_{B}\right)\right]^{(l)} \otimes C^{(\lambda)}(\hat{R})\right]^{(1)}\right\|\left(N_{A}^{\prime} N_{B}^{\prime}\right) N^{\prime} L^{\prime} ; J^{\prime}\right\rangle \\
& =(-1)^{L+N_{A}+N_{B}}\left[1, l, J, J^{\prime}, L, L^{\prime}, N, N^{\prime}, N_{A}, N_{A}^{\prime}, N_{B}, N_{B}^{\prime}\right]^{1 / 2} \\
& \times\left(\begin{array}{ccc}
N_{A} & l_{1} & N_{A}^{\prime} \\
0 & 0 & 0
\end{array}\right)\left(\begin{array}{ccc}
N_{B} & l_{2} & N_{B}^{\prime} \\
0 & 0 & 0
\end{array}\right)\left(\begin{array}{ccc}
L & \lambda & L^{\prime} \\
0 & 0 & 0
\end{array}\right)\left\{\begin{array}{ccc}
N_{A} & N_{A}^{\prime} & l_{1} \\
N_{B} & N_{B}^{\prime} & l_{2} \\
N & N^{\prime} & l
\end{array}\right\}\left\{\begin{array}{ccc}
N & N^{\prime} & l \\
L & L^{\prime} & \lambda \\
J & J^{\prime} & 1
\end{array}\right\} .
\end{aligned}
$$

${ }^{1}$ M. F. Crawford, H. L. Welsh, and J. L. Locke, Phys. Rev. 75, 1607 (1949). ${ }^{2}$ G. Birnbaum, J. Quant. Spectrosc. Radiat. Transfer 19, 51 (1978).

${ }^{3}$ M. Gustafsson, L. Frommhold, D. Bailly, J.-P. Bouanich, and C. Brodbeck, J. Chem. Phys. 119, 12264 (2003).

${ }^{4}$ W. Meyer, L. Frommhold, and G. Birnbaum, Phys. Rev. A 39, 2434 (1989).

${ }^{5}$ M. El-Kader, G. Maroulis, and E. Bich, Chem. Phys. 403, 37 (2012).

${ }^{6}$ W. Meyer, A. Borysow, and L. Frommhold, Phys. Rev. A 40, 6931 (1989).

${ }^{7}$ M. Abel, L. Frommhold, X. Li, and K. L. C. Hunt, J. Phys. Chem. A 115, 6805 (2011).

${ }^{8}$ A. R. W. McKellar and J. Schaefer, J. Chem. Phys. 95, 3081 (1991).

${ }^{9}$ M. Abel and L. Frommhold, Can. J. Phys. 91, 0532 (2013).

${ }^{10}$ G. Herzberg, Astrophys. J. 115, 337 (1952).

${ }^{11}$ T. H. Greif, V. Bromm, P. C. Clark, S. C. O. Glover, R. J. Smith, R. S. Klessen, N. Yoshida, and V. Springel, Mon. Not. R. Astron. Soc. 424, 399 (2012).

${ }^{12}$ S. T. Hodgkin, B. R. Oppenheimer, N. C. Hambly, R. F. Jameson, S. J. Smartt, and I. A. Steele, Nature 403, 57 (2000).

${ }^{13}$ T. Karman, E. Miliordos, K. L. C. Hunt, G. C. Groenenboom, and A. van der Avoird, J. Chem. Phys. 142, 084306 (2015).

${ }^{14}$ P. S. Julienne, Phys. Rev. A 26, 3299 (1982).

${ }^{15}$ P. S. Julienne and F. H. Mies, Phys. Rev. A 30, 831 (1984)

${ }^{16}$ F. H. Mies, P. S. Julienne, Y. B. Band, and S. J. Singer, J. Phys. B 19, 3249 (1986).

${ }^{17}$ P. S. Julienne and F. H. Mies, Phys. Rev. A 34, 3792 (1986).

${ }^{18}$ M. Gustafsson, L. Frommhold, and W. Meyer, J. Chem. Phys. 113, 3641 (2000).

${ }^{19}$ L. Frommhold, Collision-Induced Absorption in Gases (Cambridge Univ. Press, Cambridge, 1994).
${ }^{20} \mathrm{~K}$. Huber and G. Herzberg, Constants of Diatomic Molecules; Diatomic Constants for $\mathrm{H}_{2}$, http://webbook.nist.gov, 1976.

${ }^{21}$ M. Abramowitz and I. A. Stegun, Handbook of Mathematical Functions (National Bureau of Standards, Washington, DC, 1964), http://www.math.sfu.ca/ cbm/aands.

${ }^{22}$ D. A. Varshalovich, A. N. Moskalev, and V. K. Khersonskii, Quantum Theory of Angular Momentum (World Scientific, Singapore, 1988).

${ }^{23}$ A. Messiah, Quantum Mechanics (North Holland, Amsterdam, 1969).

${ }^{24}$ B. R. Johnson, NRCC Proc. 5, 86 (1979).

${ }^{25}$ E. Miliordos and K. L. C. Hunt, "Theoretical calculation of the second pressure, acoustic, Joule-Thomson, refractivity and dielectric virial coefficients of molecular hydrogen," J. Chem. Phys. (submitted).

${ }^{26}$ S. F. Boys and F. Bernardi, Mol. Phys. 19, 553 (1970).

${ }^{27}$ K. T. Tang and J. P. Toennies, J. Chem. Phys. 80, 3726 (1984).

${ }^{28}$ T.-S. Ho and H. Rabitz, J. Chem. Phys. 104, 2584 (1996).

${ }^{29} \mathrm{P}$. R. Bunker and P. Jensen, Molecular Symmetry and Spectroscopy, 2nd ed. (NRC Research Press, Ottawa, 1998).

${ }^{30}$ R. Martinazzo, E. Bodo, and F. Gianturco, Comput. Phys. Commun. 151, 187 (2003).

${ }^{31}$ D. R. Bosomworth and H. P. Gush, Can J. Phys. 43, 729 (1965).

${ }^{32}$ S. Cunsolo and H. P. Gush, Can. J. Phys. 50, 2058 (1972).

${ }^{33}$ I. R. Dagg, G. E. Reesor, and J. L. Urbaniak, Can. J. Phys. 53, 1764 (1975).

${ }^{34}$ Z. J. Kiss, H. P. Gush, and H. L. Welsh, Can. J. Phys. 37, 362 (1959).

${ }^{35}$ J. W. Mactaggart and J. L. Hunt, Can. J. Phys. 47, 65 (1969).

${ }^{36}$ J. P. Colpa and J. A. A. Ketelaar, Mol. Phys. 1, 14 (1958).

${ }^{37}$ J. Schäfer and W. E. Köhler, Z. Phys. D: At., Mol. Clusters 13, 217 (1989).

${ }^{38}$ J. E. Bohr and K. L. C. Hunt, J. Chem. Phys. 87, 3821 (1987). 